\title{
Estimates in Besov spaces for transport and transport-diffusion equations with almost Lipschitz coefficients
}

\section{Raphaël Danchin}

\begin{abstract}
This paper aims at giving an overview of estimates in general Besov spaces for the Cauchy problem on $t=0$ related to the vector field $\partial_{t}+v \cdot \nabla$. The emphasis is on the conservation or loss of regularity for the initial data.

When $\nabla v$ belongs to $L^{1}\left(0, T ; L^{\infty}\right)$ (plus some convenient conditions depending on the functional space considered for the data), the initial regularity is preserved. On the other hand, if $\nabla v$ is slightly less regular (e.g. $\nabla v$ belongs to some limit space for which the embedding in $L^{\infty}$ fails), the regularity may coarsen with time. Different scenarios are possible going from linear to arbitrarily small loss of regularity. This latter result will be used in a forthcoming paper to prove global well-posedness for two-dimensional incompressible density-dependent viscous fluids (see [11]).

Besides, our techniques enable us to get estimates uniformly in $\nu \geq 0$ when adding a diffusion term $-\nu \Delta u$ to the transport equation.
\end{abstract}

\section{Introduction}

This paper is concerned with estimates in Besov spaces for transport-diffusion equations:

$$
\left\{\begin{array}{l}
\partial_{t} f+v \cdot \nabla f-\nu \Delta f=g \\
f_{t=0}=f_{0}
\end{array}\right.
$$

where $\nu \geq 0$ stands for a constant diffusion parameter.

2000 Mathematics Subject Classification: 35B45, 35L45, 35Q35.

Keywords: Transport equation, transport-diffusion equation, estimates in Besov spaces, almost Lipschitz vectorfield, loss of regularity. 
Such equations appear as the result of linearization in a number of PDE's coming from fluid mechanics and have been extensively studied. In the case $\nu=0$, existence and uniqueness theory in $L^{\infty}$ has been studied under very weak assumptions on $v$ (roughly $v \in W^{1,1}$ (or even $B V$ ) with $\operatorname{div} v \in L^{\infty}$, see e.g [13], [7], [8] and the references therein).

As our work is motivated by the study of nonlinear models, we aim at estimating the fractional derivatives of the solutions to $\left(\mathcal{T}_{\nu}\right)$. It is well known that such estimates are available when $v$ has enough regularity. Roughly speaking, the regularity of the initial data is expected to be preserved as soon as $\nabla v$ belongs to $L^{1}\left(0, T ; L^{\infty}\right)$ (plus some convenient assumption depending on the number of derivatives to be transported). When $\nu=0$, this qualitative result has been proved in a number of functional frameworks:

- Sobolev spaces $H^{s}$ with $0 \leq s<\frac{N}{2}$ provided that $\nabla v \in L^{1}\left(0, T ; H^{\frac{N}{2}} \cap L^{\infty}\right)$,

- Hölder spaces $C^{r}$ if $|r| \leq 1, \nabla v \in L^{1}\left(0, T ; L^{\infty}\right)$ and $\operatorname{div} v=0$ (see e.g. [3]),

- General Besov spaces $B_{p, r}^{s}$ with $1 \leq p, r \leq+\infty$ and $-\frac{N}{p}<s<\frac{N}{p}+1$ (or $|s|<\frac{N}{p}+1$ if $\operatorname{div} v=0$ ) if $\nabla v \in L^{1}\left(0, T ; B_{p, r}^{\frac{N}{p}} \cap L^{\infty}\right.$ ) (see e.g [10]).

In section 2 of the present paper, we state estimates in $B_{p, r}^{\sigma}$ for $\left(\mathcal{T}_{\nu}\right)$ whereas $\nabla v$ belongs to some different Besov space $B_{p_{2}, r_{2}}^{\sigma^{\prime}}$ and is bounded. The main novelty is that $p_{2}$ may differ from $p$ and that the estimates do not depend on $\nu$. The reader is referred to proposition 2.1 for more details.

On the other hand, when $\nabla v$ fails to be in $L^{1}\left(0, T ; L^{\infty}\right)$, the initial regularity is unlikely to be preserved. Nevertheless, if $v$ is "almost" lipschitz with respect to the space variables then the solution $f$ may be estimated in spaces whose regularity index coarsens with time. This fact has been observed several times by different authors.

In the case of Besov spaces with third index $r=+\infty$, H. Bahouri and J.-Y. Chemin proved a linear loss of regularity under the hypothesis that $v$ is log-lipschitz:

$$
\|v\|_{L L} \stackrel{\text { def }}{=} \sup _{0<|x-y|<e^{-1}} \frac{|v(y)-v(x)|}{|y-x|(1-\log |y-x|)}<+\infty .
$$

Assuming that $|\sigma|<1$, they prove the following inequality in [1]:

$$
\|f(t)\|_{B_{p, \infty}^{\sigma_{t}}} \leq 2\left(\left\|f_{0}\right\|_{B_{p, r}^{\sigma}}+\int_{0}^{t}\|g(\tau)\|_{B_{p, \infty}^{\sigma_{\tau}}} d \tau\right)
$$

whenever $\sigma_{t} \stackrel{\text { def }}{=} \sigma-C \int_{0}^{t}\|v(\tau)\|_{L L} d \tau>-1$. 
This result has been improved in [6]. Moreover the regularity assumption on the right-hand side $g$ may be somewhat weakened (see [9]).

Let us mention in passing that results in the same spirit have been used by M. Vishik for solving incompressible Euler equations in limit Besov spaces (see [19]), and by F. Planchon to improve the Beale-Kato-Majda blow-up criterion $([17])$.

Section 3.2 of the present paper is devoted to the proof of similar estimates for $\left(\mathcal{T}_{\nu}\right)$ uniformly in $\nu$ in general Besov spaces (see theorems 3.2, 3.4, 3.9 and 3.10 below.)

It has also been observed that if $v$ is better than log-lipschitz, e.g

$$
\sup _{0<|x-y|<e^{-1}} \frac{|v(y)-v(x)|}{|y-x|\left(1-(\log |y-x|)^{\alpha}\right)}<+\infty .
$$

for some $\alpha \in(0,1)$, then the loss of regularity is arbitrarily small.

In [5], J.-Y. Chemin and N. Lerner noticed that the flow associated to a vector-field $v$ whose gradient belongs to a space slightly larger than $L^{1}\left(0, T ; H^{\frac{N}{2}}\right)$ remains in $C^{1-\epsilon}$ on $[0, T]$ for $\epsilon$ arbitrarily small despite the fact that $H^{\frac{N}{2}}$ is not embedded in $L^{\infty}$. The proof lies on the fact that $v$ satisfies an inequality of type (0.1).

In [15], B. Desjardins stated an even more accurate result in the framework of the two-dimensional torus $\mathbb{T}^{2}$. Here the flow to a vector-field $v \in$ $L^{2}\left(0, T ; H^{2}\right)$ is shown to remain in $W^{2, p}$ for all $p<2$. In the case of a bounded $N$-dimensional domain, if the symmetric part of $\nabla v$ belongs to $L^{p}\left(0, T ; W^{\frac{N}{p}, p}\right)$ with $1<p<+\infty$, and $f_{0} \in W^{1, r}$ then $f \in C\left([0, T] ; W^{1, q}\right)$ for all $q<r$ (see [14]).

Theorem 3.12 of the present paper implies the following result:

Theorem 0.1 Assume that $\nabla v \in L^{1}\left(0, T ; B_{p_{2}, r_{2}}^{\frac{N}{p_{2}}}\right)$ for some $1 \leq p_{2} \leq+\infty$ and $r_{2} \in(1,+\infty)$. Let $1 \leq p, r \leq+\infty$. Let $p^{\prime}$ be the conjugate exponent of $p$, and $\sigma$ be such that

$$
\begin{aligned}
\sigma & >-N \min \left(\frac{1}{p_{2}}, \frac{1}{p^{\prime}}\right) \quad\left(\sigma>-1-N \min \left(\frac{1}{p_{2}}, \frac{1}{p^{\prime}}\right) \quad \text { if } \quad \operatorname{div} v=0\right) \\
\text { and } \sigma & <1+\frac{N}{p_{2}} \text {. }
\end{aligned}
$$

Let $\epsilon>0$ and let $f$ solve $\left(\mathcal{T}_{\nu}\right)$. There exists $C=C\left(N, \epsilon, p, p_{2}, r_{2}, \sigma\right)$ such that the following inequality holds true on $[0, T]$ uniformly in $\nu$ :

$\|f(t)\|_{B_{p, r}^{\sigma, \epsilon}} \leq C\left(\left\|f_{0}\right\|_{B_{p, r}^{\sigma}}+\int_{0}^{t}\|g(\tau)\|_{B_{p, r}^{\sigma}} d \tau\right) \exp \left\{C\left(\int_{0}^{t}\|\nabla v(\tau)\|_{B_{p_{2}, r_{2}}^{\frac{N}{p_{2}}}} d \tau\right)^{r_{2}}\right\}$. 
We intend to use the above result to prove global well-posedness for the inhomogeneous incompressible Navier-Stokes equations in $\mathbb{T}^{2}$ or $\mathbb{R}^{2}$ (see [11]).

Remark 0.2 As our results strongly rely on the use of Fourier analysis (namely the Littlewood-Paley decomposition), we restricted ourselves to the case of $\mathbb{T}^{N}$ or $\mathbb{R}^{N}$ with $N \geq 1$. In the case $\nu=0$ however, we expect our results to be true in any smooth domain $\Omega$ if $v$ is tangent to the boundary. As a matter of fact, in the case of a Lipschitz vector-field $v$, estimates in certain Besov spaces have been proved in [16].

Remark 0.3 In order to simplify the statements, we did not track systematically the gain of derivatives induced by the diffusion operator $-\nu \Delta$. This work has been done in proposition 2.1 and leads to uniform estimates for $\nu f$ in a space very close to $L^{1}\left(0, t ; B_{p, r}^{\sigma+2}\right)$ whereas $f_{0}$ is in $B_{p, r}^{\sigma}$. A careful reading of our proofs should enable us to get a similar gain of two derivatives in the theorems of section 3.

Our paper is structured as follows. The first section is devoted to a basic presentation of Littlewood-Paley decomposition and Besov spaces. In section 2, we state general estimates in Besov spaces in the case when $v$ is Lipschitz. Section 3 is devoted to the study of the case when $v$ is not Lipschitz. We focus on the study of "abstract" coupled inequalities from which general uniform (with respect to $\nu$ ) estimates for $\left(\mathcal{T}_{\nu}\right)$ may be inferred. We then give several examples with either linear loss of derivatives or arbitrarily small loss of derivatives depending on the assumption made on $\nabla v$. An appendix is devoted to the proof of technical estimates pertaining to a commutator.

Notation: In order to have more concise statements, we shall adopt the convention that $\sigma>[-1]-\alpha_{1}$ means that $\sigma>-\alpha_{1}$ has to be satisfied for a general vector-field $v$, and that this condition may be weakened into $\sigma>-\alpha_{1}-1$ if $\operatorname{div} v=0$.

\section{Besov spaces and Littlewood-Paley decomposition}

The proof of the results presented in the paper is based on a dyadic decomposition in Fourier variables, the so-called (inhomogeneous) Littlewood-Paley decomposition. For the sake of conciseness, we only treat the case of $\mathbb{R}^{N}$. The reader is referred to [10] for a similar construction in $\mathbb{T}^{N}$.

Let $(\chi, \varphi)$ be a couple of $C^{\infty}$ functions with

$$
\begin{gathered}
\operatorname{Supp} \chi \subset\left\{|\xi| \leq \frac{4}{3}\right\}, \quad \operatorname{Supp} \varphi \subset\left\{\frac{3}{4} \leq|\xi| \leq \frac{8}{3}\right\}, \\
\forall \xi \in \mathbb{R}^{N}, \chi(\xi)+\sum_{q \in \mathbb{N}} \varphi\left(2^{-q} \xi\right)=1 .
\end{gathered}
$$


Denoting $\varphi_{q}(\xi)=\varphi\left(2^{-q} \xi\right), h_{q}=\mathcal{F}^{-1} \varphi_{q}$ and $\breve{h}=\mathcal{F}^{-1} \chi$, we define the dyadic blocks as

$$
\begin{gathered}
\Delta_{q} u \stackrel{\text { def }}{=} 0 \quad \text { if } \quad q \leq-1, \quad \Delta_{-1} u \stackrel{\text { def }}{=} \chi(D) u=\int_{\mathbb{R}^{N}} \check{h}(y) u(x-y) d y, \\
\Delta_{q} u \stackrel{\text { def }}{=} \varphi\left(2^{-q} D\right) u=\int_{\mathbb{R}^{N}} h_{q}(y) u(x-y) d y \quad \text { if } \quad q \geq 0 .
\end{gathered}
$$

We shall also use the following low-frequency cut-off:

$$
S_{q} u \stackrel{\text { def }}{=} \sum_{k \leq q-1} \Delta_{k} u=\chi\left(2^{-q} D\right) u
$$

One can easily prove that

$$
\forall u \in \mathcal{S}^{\prime}\left(\mathbb{R}^{N}\right), \quad u=\sum_{q \in \mathbb{Z}} \Delta_{q} u
$$

Littlewood-Paley decomposition has nice properties of quasi-orthogonality:

$$
\Delta_{k} \Delta_{q} u \equiv 0 \quad \text { if } \quad|k-q| \geq 2 \text { and } \Delta_{k}\left(S_{q-1} u \Delta_{q} u\right) \equiv 0 \quad \text { if } \quad|k-q| \geq 5
$$

Let us now define the (non-homogeneous) Besov spaces:

Definition 1.1 For $s \in \mathbb{R},(p, r) \in[1,+\infty]^{2}$ and $u \in \mathcal{S}^{\prime}\left(\mathbb{R}^{N}\right)$, we set $\|u\|_{B_{p, r}^{s}} \stackrel{\text { def }}{=}\left(\sum_{q \geq-1} 2^{r s q}\left\|\Delta_{q} u\right\|_{L^{p}}^{r}\right)^{\frac{1}{r}}$ if $r<+\infty$ and $\|u\|_{B_{p, \infty}^{s}} \stackrel{\text { def }}{=} \sup _{q \geq-1} 2^{s q}\left\|\Delta_{q} u\right\|_{L^{p}}$.

We then define the Besov space $B_{p, r}^{s}$ as the set of temperate distributions with finite $\|\cdot\|_{B_{p, r}^{s}}$ norm.

The definition of $B_{p, r}^{s}$ does not depend on the choice of the couple $(\chi, \varphi)$. One can further remark that $H^{s}$ coincide with $B_{2,2}^{s}$, and that $C^{r}=B_{\infty, \infty}^{r}$ if $r \in \mathbb{R}^{+} \backslash \mathbb{N}$.

The reader is referred to [18] for a complete study of Besov spaces. Let us just recall some of their most basic properties.

Proposition 1.2 The following properties hold:

i) Derivatives: we have $\|\nabla u\|_{B_{p, r}^{s-1}} \lesssim\|u\|_{B_{p, r}^{s}}$.

ii) Sobolev embeddings:

- If $p_{1} \leq p_{2}$ and $r_{1} \leq r_{2}$ then $B_{p_{1}, r_{1}}^{s} \hookrightarrow B_{p_{2}, r_{2}}^{s-N\left(\frac{1}{p_{1}}-\frac{1}{p_{2}}\right)}$. 
- If $s_{1}>s_{2}$ and $1 \leq p, r_{1}, r_{2} \leq+\infty$, then $B_{p, r_{1}}^{s_{1}} \hookrightarrow B_{p, r_{2}}^{s_{2}}$.

- If $1 \leq p \leq+\infty$ then $B_{p, 1}^{\frac{N}{p}} \hookrightarrow L^{\infty}$.

iii) Algebraic properties: for $s>0, B_{p, r}^{s} \cap L^{\infty}$ is an algebra.

iv) Real interpolation: $\left(B_{p, r}^{s_{1}}, B_{p, r}^{s_{2}}\right)_{\theta, r^{\prime}}=B_{p, r^{\prime}}^{\theta s_{2}+(1-\theta) s_{1}}$.

We aim at proving estimates for $\left(\mathcal{T}_{\nu}\right)$ in spaces $L^{\rho}\left(0, T ; B_{p, r}^{\sigma}\right)$. Taking into account the definition of Besov spaces, it is natural to localize the equations through Littlewood-Paley decomposition. We then get estimates for each dyadic block and perform integration in time. But, in doing so, we obtain bounds in spaces which are not of type $L^{\rho}\left(0, T ; B_{p, r}^{s}\right)$. That remark naturally leads to the following definition (introduced in [4]):

Definition 1.3 Let $s \in \mathbb{R}, 1 \leq p, r, \rho \leq+\infty$ and $T \in[0,+\infty]$. We set

$$
\|u\|_{\widetilde{L}_{T}^{\rho}\left(B_{p, r}^{s}\right)} \stackrel{\text { def }}{=}\left(\left(\int_{0}^{T}\left\|\Delta_{-1} u(t)\right\|_{L^{p}}^{\rho} d t\right)^{\frac{r}{\rho}}+\sum_{q \in \mathbb{N}} 2^{r q s}\left(\int_{0}^{T}\left\|\Delta_{q} u(t)\right\|_{L^{p}}^{\rho} d t\right)^{\frac{r}{\rho}}\right)^{\frac{1}{r}}
$$

and denote by $\widetilde{L}_{T}^{\rho}\left(B_{p, r}^{s}\right)$ the set of distributions of $\mathcal{S}^{\prime}\left(0, T \times \mathbb{R}^{N}\right)$ with finite $\|\cdot\|_{\widetilde{L}_{T}^{\rho}\left(B_{p, r}^{s}\right)}$ norm.

Let us remark that by virtue of Minkowski inequality, we have

$$
\|u\|_{\widetilde{L}_{T}^{\rho}\left(B_{p, r}^{s}\right)} \leq\|u\|_{L_{T}^{\rho}\left(B_{p, r}^{s}\right)} \quad \text { if } \rho \leq r
$$

and

$$
\|u\|_{L_{T}^{\rho}\left(B_{p, r}^{s}\right)} \leq\|u\|_{\widetilde{L}_{T}^{\rho}\left(B_{p, r}^{s}\right)} \quad \text { if } \rho \geq r .
$$

\section{A priori estimates with no loss of regularity}

We first concentrate on the case when $\nabla v$ belongs to $L^{1}\left(0, T ; L^{\infty}\right)$. In this case, the initial Besov regularity is conserved:

Proposition 2.1 Let $1 \leq p, p_{2}, r \leq+\infty$ and $p^{\prime} \stackrel{\text { def }}{=}(1-1 / p)^{-1}$. Assume that

$$
\sigma>[-1]-N \min \left(\frac{1}{p_{2}}, \frac{1}{p^{\prime}}\right)
$$

Denote $f^{H F} \stackrel{\text { def }}{=} f-\Delta_{-1} f$. There exists a constant $C$ depending on $N, p, p_{2}$ and $\sigma$ but not on $\nu$ and a universal constant $\kappa>0$ such that the following 
estimates hold:

$$
\begin{aligned}
\|f\|_{\widetilde{L}_{t}^{\infty}\left(B_{p, r}^{\sigma}\right)}+\kappa \nu & \left(\frac{p-1}{p^{2}}\right)\left\|f^{H F}\right\|_{\widetilde{L}_{t}^{1}\left(B_{p, r}^{\sigma+2}\right)} \\
& \leq\left(\left\|f_{0}\right\|_{B_{p, r}^{\sigma}}+\int_{0}^{t} e^{-C Z(\tau)}\|g(\tau)\|_{B_{p, r}^{\sigma}} d \tau\right) e^{C Z(t)}
\end{aligned}
$$

$$
\|f\|_{\widetilde{L}_{t}^{\infty}\left(B_{p, r}^{\sigma}\right)}+\kappa \nu\left(\frac{p-1}{p^{2}}\right)\left\|f^{H F}\right\|_{\widetilde{L}_{t}^{1}\left(B_{p, r}^{\sigma+2}\right)} \leq\left(\left\|f_{0}\right\|_{B_{p, r}^{\sigma}}+\|g\|_{\widetilde{L}_{t}^{1}\left(B_{p, r}^{\sigma}\right)}\right) e^{C Z(t)},
$$

with

$$
\left\{\begin{array}{l}
Z(t)=\int_{0}^{t}\|\nabla v(\tau)\|_{B_{p_{2}, \infty}^{\frac{N}{p_{2}}} \cap L^{\infty}} d \tau \quad \text { if } \sigma<1+\frac{N}{p_{2}}, \\
Z(t)=\int_{0}^{t}\|\nabla v(\tau)\|_{B_{p_{2}, r}^{\sigma-1}} d \tau \quad \text { if } \sigma>1+\frac{N}{p_{2}} \text { or }\left\{\sigma=1+\frac{N}{p_{2}} \text { and } r=1\right\} .
\end{array}\right.
$$

If $f=v$ then for all $\sigma>0(\sigma>-1$ if $\operatorname{div} v=0)$ estimates (2.1) and (2.2) hold with

$$
Z(t)=\int_{0}^{t}\|\nabla v(\tau)\|_{L^{\infty}} d \tau
$$

Proof: Applying the operator $\Delta_{q}$ to $\left(\mathcal{T}_{\nu}\right)$ yields

$$
\left\{\begin{array}{l}
\left(\partial_{t}+S_{q+1} v \cdot \nabla\right) \Delta_{q} f-\nu \Delta \Delta_{q} f=\Delta_{q} F+R_{q} \\
\Delta_{q} f_{\mid t=0}=\Delta_{q} f_{0}
\end{array}\right.
$$

with $R_{q} \stackrel{\text { def }}{=} S_{q+1} v \cdot \nabla \Delta_{q} f-\Delta_{q}(v \cdot \nabla f)$.

As $S_{q+1} v$ is smooth, one readily gets in the case $\nu=0$ :

$$
\begin{aligned}
\left\|\Delta_{q} f(t)\right\|_{L^{p}} & \leq\left\|\Delta_{q} f_{0}\right\|_{L^{p}}+\int_{0}^{t}\left(\left\|R_{q}(\tau)\right\|_{L^{p}}+\frac{1}{p}\left\|\operatorname{div} S_{q+1} v(\tau)\right\|_{L^{\infty}}\left\|\Delta_{q} f(\tau)\right\|_{L^{p}}\right) d \tau \\
& (2.3) \\
& +\int_{0}^{t}\left\|\Delta_{q} F(\tau)\right\|_{L^{p}} d \tau,
\end{aligned}
$$

where it is understood that $1 / p=0$ if $p=+\infty$.

Let us now focus on the case $\nu>0$ and $q \geq 0$.

1. Case $1<p<+\infty$.

After multiplying $\left(\mathcal{T}_{q}\right)$ by $\left|\Delta_{q} f\right|^{p-1} \operatorname{sgn}\left(\Delta_{q} f\right)$, the new term we have to deal with is

$$
-\nu \int \Delta_{q} f\left|\Delta_{q} f\right|^{p-1} \operatorname{sgn}\left(\Delta_{q} f\right) d x .
$$


As $q \geq 0$ (hence $\mathcal{F} \Delta_{q} f$ is supported in $C\left(0, \frac{3}{4} 2^{q}, \frac{8}{3} 2^{q}\right)$ ), lemma A.5 in [12] ensures that, for some universal constant $\kappa>0$,

$$
-\int \Delta_{q} f\left|\Delta_{q} f\right|^{p-1} \operatorname{sgn}\left(\Delta_{q} f\right) d x \geq \kappa\left(\frac{p-1}{p^{2}}\right) 2^{2 q} \int\left|\Delta_{q} f\right|^{p} d x,
$$

hence

$$
\begin{aligned}
\left\|\Delta_{q} f(t)\right\|_{L^{p}}+ & \kappa \nu\left(\frac{p-1}{p^{2}}\right) 2^{2 q} \int_{0}^{t}\left\|\Delta_{q} f(\tau)\right\|_{L^{p}} d \tau \leq \\
\leq\left\|\Delta_{q} f_{0}\right\|_{L^{p}} & +\int_{0}^{t}\left(\left\|R_{q}(\tau)\right\|_{L^{p}}+\frac{1}{p}\left\|\operatorname{div} S_{q+1} v(\tau)\right\|_{L^{\infty}}\left\|\Delta_{q} f(\tau)\right\|_{L^{p}}\right) d \tau \\
& +\int_{0}^{t}\left\|\Delta_{q} F(\tau)\right\|_{L^{p}} d \tau .
\end{aligned}
$$

2. Case $p=1$.

The same estimate holds true. Indeed, introduce the function

$$
T_{\epsilon}(x) \stackrel{\text { def }}{=} x / \sqrt{\epsilon^{2}+x^{2}} .
$$

From a straightforward integration by parts, the following inequality is inferred:

$$
-\int \Delta \Delta_{q} u T_{\epsilon}^{\prime}\left(\Delta_{q} u\right) d x=\int \frac{\epsilon^{2}\left|\nabla \Delta_{q} u\right|^{2}}{\epsilon^{2}+\left|\Delta_{q} u\right|^{2}} d x \geq 0
$$

Hence, multiplying equation $\left(\mathcal{T}_{q}\right)$ by $T_{\epsilon}\left(\Delta_{q} f\right)$, integrating in space and in time and using Lebesgue dominated convergence theorem to pass to the limit $\epsilon \rightarrow 0$ eventually yields inequality (2.5).

3. Case $p=+\infty$. It stems from the maximum principle.

That the term induced by $-\nu \Delta \Delta_{q} f$ is also non-negative in the case $q=-1$ is left to the reader. This entails that

$$
\begin{aligned}
\left\|\Delta_{1} f(t)\right\|_{L^{p}} \leq & \left\|\Delta_{1} f_{0}\right\|_{L^{p}}+\int_{0}^{t}\left(\left\|R_{-1}(\tau)\right\|_{L^{p}}+\frac{1}{p}\left\|\operatorname{div} S_{0} v(\tau)\right\|_{L^{\infty}}\left\|\Delta_{1} f(\tau)\right\|_{L^{p}}\right) d \tau \\
& +\int_{0}^{t}\left\|\Delta_{-1} F(\tau)\right\|_{L^{p}} d \tau .
\end{aligned}
$$

Let us admit the following lemma, the proof of which is postponed in appendix: 
Lemma 2.2 Let $\sigma \in \mathbb{R}$ and $1 \leq p \leq p_{2} \leq+\infty$. Let $p_{1}=\left(1 / p-1 / p_{2}\right)^{-1}$. There exists a constant $K=K(N)$ such that

$$
\begin{aligned}
2^{q \sigma}\left\|R_{q}\right\|_{L^{p}} \leq & 4^{|\sigma|} K\left(\sum_{\left|q^{\prime}-q\right| \leq 4}\left(\left\|\Delta_{-1} \nabla v\right\|_{L^{\infty}}+\left\|S_{q^{\prime}-1} \nabla v\right\|_{L^{\infty}}\right) 2^{q^{\prime} \sigma}\left\|\Delta_{q^{\prime}} f\right\|_{L^{p}}\right. \\
& +\sum_{\substack{q^{\prime} \geq q-3 \\
+}} 2^{q-q^{\prime}} 2^{q \sigma}\left\|\Delta_{q} f\right\|_{L^{p}}\left\|\Delta_{q^{\prime}} \nabla v\right\|_{L^{\infty}} \\
& +\sum_{\substack{\left|q^{\prime}-q\right| \leq 4 \\
q^{\prime \prime} \leq q^{\prime}-2}} 2^{\left(q-q^{\prime \prime}\right)\left(\sigma-1-\frac{N}{p_{2}}\right)} 2^{q^{\prime \prime} \sigma}\left\|\Delta_{q^{\prime \prime}} f\right\|_{L^{p}} 2^{q^{\prime} \frac{N}{p_{2}}}\left\|\Delta_{q^{\prime}} \nabla v\right\|_{L^{p_{2}}} \\
+ & \sum_{\substack{q^{\prime} \geq q-3 \\
\left|q^{\prime}-q^{\prime \prime}\right| \leq 1}} 2^{\left(q-q^{\prime}\right)}\left(\sigma+N \min \left(\frac{1}{p_{2}}, \frac{1}{p^{\prime}}\right)\right)_{\times} \\
& \left.\quad \times 2^{q^{\prime} \frac{N}{p_{2}}}\left(2^{q-q^{\prime}}\left\|\Delta_{q^{\prime}} \nabla v\right\|_{L^{p_{2}}}+\left\|\Delta_{q^{\prime}} \operatorname{div} v\right\|_{L^{p_{2}}}\right) 2^{q^{\prime \prime} \sigma}\left\|\Delta_{q^{\prime \prime}} f\right\|_{L^{p}}\right) .
\end{aligned}
$$

Besides, the third term in the right-hand side may be replaced by

$$
16^{|\sigma|} K\|\nabla f\|_{L^{p_{1}}} \sum_{\left|q^{\prime}-q\right| \leq 4} 2^{q^{\prime}(\sigma-1)}\left\|\Delta_{q^{\prime}} \nabla v\right\|_{L^{p_{2}}} .
$$

Let $\kappa_{p} \stackrel{\text { def }}{=} \kappa(p-1) / p^{2}, f_{q} \stackrel{\text { def }}{=} 2^{q \sigma}\left\|\Delta_{q} f\right\|_{L^{p}}$ and $g_{q} \stackrel{\text { def }}{=} 2^{q \sigma}\left\|\Delta_{q} g\right\|_{L^{p}}$. Assume that $\sigma<1+N / p_{2}$ and denote for a suitably large $K=K(N)$

$$
\left\{\begin{aligned}
& v_{q} \stackrel{\text { def }}{=} K(\left\|\Delta_{-1} \nabla v\right\|_{L^{\infty}}+\left\|S_{q} \nabla v\right\|_{L^{\infty}} \\
&\left.+\sum_{i \in \mathbb{N}} 2^{-i}\left\|\Delta_{q+i} \nabla v\right\|_{L^{\infty}}+\sum_{\left|q^{\prime}-q\right| \leq 4} 2^{q^{\prime} \frac{N}{p_{2}}}\left\|\Delta_{q^{\prime}} \nabla v\right\|_{L^{p_{2}}}\right) \\
& w_{q} \stackrel{\text { def }}{=} z_{q} \stackrel{\text { def }}{=} K \sum_{\left|q^{\prime}-q\right| \leq 4} 2^{q^{\prime} \frac{N}{p_{2}}}\left\|\Delta_{q^{\prime}} \nabla v\right\|_{L^{p_{2}}} .
\end{aligned}\right.
$$

Further denote

(2.10) $\sigma_{2} \stackrel{\text { def }}{=} 1+\frac{N}{p_{2}}-\sigma \quad$ and $\sigma_{1} \stackrel{\text { def }}{=} \begin{cases}\sigma+\min \left(\frac{N}{p_{2}}, \frac{N}{p^{\prime}}\right) & \text { if } \operatorname{div} v \neq 0, \\ 1+\sigma+\min \left(\frac{N}{p_{2}}, \frac{N}{p^{\prime}}\right) & \text { if } \operatorname{div} v=0 .\end{cases}$

Then inserting inequality (2.7) into (2.5) and (2.6) yields

$$
\begin{aligned}
& f_{q}(t)+\kappa_{p} \delta_{q} \nu 2^{2 q} \int_{0}^{t} f_{q}(\tau) d \tau \leq f_{q}(0)+\int_{0}^{t} g_{q}(\tau) d \tau+\sum_{\left|q^{\prime}-q\right| \leq 4} \int_{0}^{t} v_{q^{\prime}}(\tau) f_{q^{\prime}}(\tau) d \tau \\
& (2.11)+\sum_{q^{\prime}>q+4} \int_{0}^{t} 2^{\sigma_{1}\left(q-q^{\prime}\right)} w_{q^{\prime}}(\tau) f_{q^{\prime}}(\tau) d \tau+\sum_{q^{\prime}<q-4} \int_{0}^{t} 2^{\sigma_{2}\left(q^{\prime}-q\right)} z_{q}(\tau) f_{q^{\prime}}(\tau) d \tau,
\end{aligned}
$$

with $\delta_{-1}=0$ and $\delta_{q}=1$ if $q \geq 0$. 
With our assumptions on $\nabla v$, we have for a suitably large $K$ :

$$
\max \left(v_{q}, w_{q}, z_{q}\right) \leq a \stackrel{\text { def }}{=} K\|\nabla v\|_{B_{p_{2}, \infty}^{\frac{N}{p_{2}}} \cap L^{\infty}} .
$$

Moreover $f_{q}(t)$ may be replaced by $\sup _{\tau \in[0, t]} f_{q}(\tau)$ in the left-hand side.

Hence, taking advantage of $\sigma_{1}, \sigma_{2}>0$ for using convolution inequalities and applying, where needed, Minkowski inequality, one ends up with

$$
\begin{aligned}
\|f\|_{\widetilde{L}_{t}^{\infty}\left(B_{p, r}^{\sigma}\right)} & +\kappa_{p} \nu\left\|f^{H F}\right\|_{\widetilde{L}_{t}^{1}\left(B_{p, r}^{\sigma+2}\right)} \leq \\
& \leq\left\|f_{0}\right\|_{B_{p, r}^{\sigma}}+\int_{0}^{t} a(\tau)\|f\|_{\widetilde{L}_{\tau}^{\infty}\left(B_{p, r}^{\sigma}\right)} d \tau+\left\{\begin{array}{l}
\|g\|_{\widetilde{L}_{t}^{1}\left(B_{p, r}^{\sigma}\right)} \\
\int_{0}^{t}\|g(\tau)\|_{B_{p, r}^{\sigma}} d \tau
\end{array}\right.
\end{aligned}
$$

so that Gronwall lemma completes the proof of proposition 2.1 in the case $\sigma<1+N / p_{2}$.

The case $\sigma>1+N / p_{2}$ or $\left\{\sigma=1+N / p_{2}\right.$ and $\left.r=1\right\}$ is left to the reader. It is based on inequality (2.8) and embeddings $B_{p, r}^{\sigma-1} \hookrightarrow B_{p, 1}^{\frac{N}{p_{2}}} \hookrightarrow L^{p_{1}}$. The additional estimate in the case $f=v$ is inferred from remark A.1.

\section{Losing estimates}

The key point in the proof of proposition 2.1 is that $\nabla v$ belongs to

$$
L^{1}\left(0, T ; B_{p_{2}, \infty}^{\frac{N}{p_{2}}} \cap L^{\infty}\right) .
$$

In the present section, we make slightly weaker assumptions on $v$. The price to pay is a possible loss of derivatives in the estimates.

Throughout this section, the regularity index $\sigma$ for $f$ satisfies (2.10) (hence in particular $|\sigma| \leq 1+N$ ).

\subsection{A general statement}

Note that no regularity assumptions on $v$ are needed to get (2.11). This induces us to study the following type of coupled inequalities (we set $\kappa_{p} \nu$ to 0 to simplify the presentation):

$$
\begin{gathered}
f_{q}(t) \leq f_{q}(0)+\int_{0}^{t} g_{q}(\tau) d \tau+\int_{0}^{t} \sum_{\left|q^{\prime}-q\right| \leq N_{0}} v_{q^{\prime}}(\tau) f_{q^{\prime}}(\tau) d \tau \\
+\int_{0}^{t}\left(\sum_{q^{\prime}>q+M} 2^{\sigma_{1}\left(q-q^{\prime}\right)} w_{q^{\prime}}(\tau) f_{q^{\prime}}(\tau)\right) d \tau+\int_{0}^{t} z_{q}(\tau)\left(\sum_{q^{\prime}<q-P} 2^{\sigma_{2}\left(q^{\prime}-q\right)} f_{q^{\prime}}(\tau)\right) d \tau
\end{gathered}
$$

whenever $q \in \mathbb{Z}$ and $t \in[0, T]$. Above, $N_{0}, M$ and $P$ belong to $\mathbb{N}$. 
We further assume that

$$
v_{q}(t) \leq v_{q}^{1}(t)+v_{q}^{2}(t), \quad w_{q}(t) \leq w_{q}^{1}(t)+w_{q}^{2}(t), \quad z_{q}(t) \leq z_{q}^{1}(t)+z_{q}^{2}(t)
$$

for some measurable and nonnegative functions $v_{q}^{1}, w_{q}^{1}$, etc. on $[0, T]$.

Before stating estimates pertaining to (3.1), let us introduce a few notation:

$$
V_{q}^{1}(t) \stackrel{\text { def }}{=} \int_{0}^{t} v_{q}^{1}(\tau) d \tau, \quad W_{q}^{1}(t) \stackrel{\text { def }}{=} \int_{0}^{t} w_{q}^{1}(\tau) d \tau, \quad Z_{q}^{1}(t) \stackrel{\text { def }}{=} \int_{0}^{t} z_{q}^{1}(\tau) d \tau .
$$

For $R \in \mathbb{Z}$, let $v^{R}(t) \stackrel{\text { def }}{=} \sup _{q \leq R} v_{q}^{2}(t)$ and $V^{R}(t) \stackrel{\text { def }}{=} \int_{0}^{t} v^{R}(\tau) d \tau$. Further denote $v^{\infty}(t) \stackrel{\text { def }}{=} \sup _{q \in \mathbb{Z}} v_{q}^{2}(t)$ and $V^{\infty}(t) \stackrel{\text { def }}{=} \int_{0}^{t} v^{\infty}(\tau) d \tau$.

Define in the same way the functions $w^{R}$ and $W^{R}$ (resp. $z^{R}$ and $Z^{R}$ ) pertaining to $w^{2}$ (resp. $z^{2}$ ). Finally, let $a \wedge b$ stand for $\max (a, b)$.

For $\left(f_{q}\right)_{q \in \mathbb{Z}}$ satisfying the coupled inequalities (3.1), we have:

Proposition 3.1 Let $\kappa \geq 0$ and $\lambda>\lambda_{0}>0$. Assume that the following conditions are fulfilled for all $t \in[0, T]$ :

1. There exist $R_{1}, R_{2}$ and $R_{3}$ in $\mathbb{Z} \cup\{+\infty\}$ such that

$$
\left\{\begin{array}{l}
\text { if } R_{1}<+\infty, \quad \int_{0}^{t} v_{q}^{2}(\tau) d \tau \leq \lambda^{-1} \quad \text { for } \quad q>R_{1}, \\
\text { if } R_{1}=+\infty, \quad V^{\infty}(t)<+\infty,
\end{array}\right.
$$

and similar conditions for $w_{q}^{2}$ with $R_{2}$, and for $z_{q}^{2}$ with $R_{3}$.

2. There exist $Q_{1}, Q_{2}$ and $Q_{3}$ in $\mathbb{Z}$ such that

$$
\begin{aligned}
v_{q}^{1}(t) & \leq v_{Q_{1}}^{1}(t) \quad \text { for } q \leq Q_{1}, \\
w_{q}^{1}(t) & \leq w_{Q_{2}}^{1}(t) \text { for } q \leq Q_{2}, \\
z_{q}^{1}(t) & \leq z_{Q_{3}}^{1}(t) \quad \text { for } q \leq Q_{3} .
\end{aligned}
$$

3. Let $A_{m} \stackrel{\text { def }}{=} V_{m \wedge Q_{1}}^{1}+W_{m \wedge Q_{2}}^{1}+Z_{m \wedge Q_{3}}^{1}, \quad A_{q, q^{\prime}}^{\lambda}(t) \stackrel{\text { def }}{=} \sup _{\tau \in[0, t]}\left(e^{\lambda\left(A_{q^{\prime}}-A_{q}\right)(\tau)}\right)$,

$$
\begin{aligned}
& K_{\lambda}^{1}(t)=\max \left(\sup _{q} \sum_{q^{\prime}>q+M} 2^{\sigma_{1}\left(q-q^{\prime}\right)} A_{q, q^{\prime}}^{\lambda}(t), \sup _{q^{\prime}} \sum_{q<q^{\prime}-M} 2^{\sigma_{1}\left(q-q^{\prime}\right)} A_{q, q^{\prime}}^{\lambda}(t)\right), \\
& K_{\lambda}^{2}(t)=\max \left(\sup _{q} \sum_{q^{\prime}<q-P} 2^{\sigma_{2}\left(q^{\prime}-q\right)} A_{q, q^{\prime}}^{\lambda}(t), \sup _{q^{\prime}} \sum_{q>q^{\prime}+P} 2^{\sigma_{2}\left(q^{\prime}-q\right)} A_{q, q^{\prime}}^{\lambda}(t)\right) .
\end{aligned}
$$

Then we assume that

$$
2\left(K_{\lambda}^{1}(t)+K_{\lambda}^{2}(t)+2^{\kappa}\left(2 N_{0}+1\right)\right) \leq \lambda_{0} .
$$


4. For all $\left(q, q^{\prime}\right)$ such that $\left|q-q^{\prime}\right| \leq N_{0}$, we have

$$
\lambda\left|A_{q^{\prime}}(t)-A_{q}(t)\right| \leq \kappa \log 2
$$

Let

$$
B \stackrel{\text { def }}{=} V^{R_{1}}+W^{R_{2}}+Z^{R_{3}}, \quad \tilde{f}_{q}^{\lambda}(\tau) \stackrel{\text { def }}{=} e^{-\lambda\left(A_{q}+B\right)(\tau)} f_{q}(\tau)
$$

and

$$
\widetilde{g}_{q}^{\lambda}(\tau) \stackrel{\text { def }}{=} e^{-\lambda\left(A_{q}+B\right)(\tau)} g_{q}(\tau) .
$$

The following estimate holds true for all $r \in[1,+\infty]$ and $t \in[0, T]$ :

$$
\left(\sum_{q}\left(\sup _{\tau \in[0, t]} \tilde{f}_{q}^{\lambda}(\tau)\right)^{r}\right)^{\frac{1}{r}} \leq \frac{\lambda}{\lambda-\lambda_{0}}\left(\left(\sum_{q}\left(f_{q}(0)\right)^{r}\right)^{\frac{1}{r}}+\left(\sum_{q}\left(\int_{0}^{t} \widetilde{g}_{q}^{\lambda}(\tau) d \tau\right)^{r}\right)^{\frac{1}{r}}\right) .
$$

Proof: According to (3.1), we have for all $t \in[0, T]$ :

$$
\begin{gathered}
e^{\lambda\left(A_{q}+B\right)(t)} \widetilde{f}_{q}^{\lambda}(t) \leq f_{q}(0)+\int_{0}^{t} e^{\lambda\left(A_{q}+B\right)(\tau)} \widetilde{g}_{q}^{\lambda}(\tau) d \tau \\
+\sum_{\left|q^{\prime}-q\right| \leq N_{0}}\left(\int_{0}^{t} v_{q^{\prime}}^{1}(\tau) e^{\lambda\left(A_{q^{\prime}}+B\right)(\tau)} \widetilde{f}_{q^{\prime}}^{\lambda}(\tau) d \tau+\int_{0}^{t} v_{q^{\prime}}^{2}(\tau) e^{\lambda\left(A_{q^{\prime}}+B\right)(\tau)} \widetilde{f}_{q^{\prime}}^{\lambda}(\tau) d \tau\right)
\end{gathered}
$$$$
+\sum_{q^{\prime}>q+M} 2^{\sigma_{1}\left(q-q^{\prime}\right)}\left(\int_{0}^{t} w_{q^{\prime}}^{1}(\tau) e^{\lambda\left(A_{q^{\prime}}+B\right)(\tau)} \widetilde{f}_{q^{\prime}}^{\lambda}(\tau) d \tau+\int_{0}^{t} w_{q^{\prime}}^{2}(\tau) e^{\lambda\left(A_{q^{\prime}}+B\right)(\tau)} \widetilde{f}_{q^{\prime}}^{\lambda}(\tau) d \tau\right)
$$$$
+\sum_{q^{\prime}<q-P} 2^{\sigma_{2}\left(q^{\prime}-q\right)}\left(\int_{0}^{t} z_{q}^{1}(\tau) e^{\lambda\left(A_{q^{\prime}}+B\right)(\tau)} \widetilde{f}_{q^{\prime}}^{\lambda}(\tau) d \tau+\int_{0}^{t} z_{q}^{2}(\tau) e^{\lambda\left(A_{q^{\prime}}+B\right)(\tau)} \widetilde{f}_{q^{\prime}}^{\lambda}(\tau) d \tau\right) .
$$

According to assumption 2, we have for all $q \in \mathbb{Z}$ and $t \in[0, T]$,

$$
v_{q}^{1}(t) \leq v_{q \wedge Q_{1}}^{1}(t), \quad w_{q}^{1}(t) \leq w_{q \wedge Q_{2}}^{1}(t) \quad \text { and } \quad z_{q}^{1}(t) \leq z_{q \wedge Q_{3}}^{1}(t),
$$

whence

$$
\left\{\begin{array}{l}
\int_{0}^{t} v_{q^{\prime}}^{1}(\tau) e^{\lambda\left(A_{q^{\prime}}+B\right)(\tau)} d \tau \leq \frac{e^{\lambda\left(A_{q^{\prime}}+B\right)(t)}}{\lambda} \\
\int_{0}^{t} w_{q^{\prime}}^{1}(\tau) e^{\lambda\left(A_{q^{\prime}}+B\right)(\tau)} d \tau \leq \frac{e^{\lambda\left(A_{q^{\prime}}+B\right)(t)}}{\lambda} .
\end{array}\right.
$$

On the other hand, we have for $q^{\prime}<q-P$,

$$
\begin{aligned}
\int_{0}^{t} z_{q}^{1}(\tau) e^{\lambda\left(A_{q^{\prime}}+B\right)(\tau)} d \tau & \leq \int_{0}^{t} z_{q \wedge Q_{3}}^{1}(\tau) e^{\lambda\left(A_{q}+B\right)(\tau)} e^{\lambda\left(A_{q^{\prime}}-A_{q}\right)(\tau)} d \tau \\
& \leq \lambda^{-1} e^{\lambda\left(A_{q}+B\right)(t)} A_{q, q^{\prime}}^{\lambda}(t) .
\end{aligned}
$$


By mean of explicit integration, we get

$$
\int_{0}^{t} v_{q^{\prime}}^{2}(\tau) e^{\lambda\left(A_{q^{\prime}}(\tau)+B(\tau)\right)} d \tau \leq \begin{cases}\lambda^{-1} e^{\lambda\left(A_{q^{\prime}}(t)+B(t)\right)} & \text { if } q^{\prime} \leq R_{1} \\ \left(\int_{0}^{t} v_{q^{\prime}}^{2}(\tau) d \tau\right) e^{\lambda\left(A_{q^{\prime}}(t)+B(t)\right)} & \text { if } q^{\prime}>R_{1}\end{cases}
$$

Similar inequalities hold for $\int_{0}^{t} w_{q^{\prime}}^{2}(\tau) \exp \left(\lambda\left(A_{q}(\tau)+B(\tau)\right)\right) d \tau$, and

$$
\int_{0}^{t} z_{q}^{2}(\tau) e^{\lambda\left(A_{q^{\prime}}(\tau)+B(\tau)\right)} d \tau \leq \begin{cases}\lambda^{-1} e^{\lambda\left(A_{q^{\prime}}(t)+B(t)\right)} & \text { if } q \leq R_{3} \\ \left(\int_{0}^{t} z_{q}^{2}(\tau) d \tau\right) e^{\lambda\left(A_{q^{\prime}}(t)+B(t)\right)} & \text { if } q>R_{3}\end{cases}
$$

Let $\widetilde{F}_{q}^{\lambda}(t) \stackrel{\text { def }}{=} \sup _{\tau \in[0, t]} \widetilde{f}_{q}^{\lambda}(\tau)$. Plugging (3.4), (3.5), (3.6) and (3.7) in (3.3) and using 1 yields

$$
\begin{aligned}
\widetilde{f}_{q}^{\lambda}(t) \leq f_{q}(0) & +\int_{0}^{t} \widetilde{g}_{q}^{\lambda}(\tau) d \tau+\frac{2}{\lambda} \sum_{\left|q^{\prime}-q\right| \leq N_{0}} A_{q, q^{\prime}}^{\lambda}(t) \widetilde{F}_{q^{\prime}}^{\lambda}(t) \\
& +\frac{2}{\lambda} \sum_{q^{\prime}>q+M} 2^{\sigma_{1}\left(q-q^{\prime}\right)} A_{q, q^{\prime}}^{\lambda}(t) \widetilde{F}_{q^{\prime}}^{\lambda}(t)+\frac{2}{\lambda} \sum_{q^{\prime}<q-P} 2^{\sigma_{2}\left(q^{\prime}-q\right)} \widetilde{F}_{q^{\prime}}^{\lambda}(t) A_{q, q^{\prime}}^{\lambda}(t) .
\end{aligned}
$$

Clearly, $t$ may be replaced by any $t^{\prime} \in[0, t]$ in the left-hand side so that $\widetilde{f}_{q}^{\lambda}(t)$ may be replaced by $\widetilde{F}_{q}^{\lambda}(t)$. Now, Schur's lemma yields

$$
\begin{aligned}
\left(\sum_{q}\left(\widetilde{F}_{q}^{\lambda}(t)\right)^{r}\right)^{\frac{1}{r}} \leq & \left(\sum_{q}\left(f_{q}(0)\right)^{r}\right)^{\frac{1}{r}}+\left(\sum_{q}\left(\int_{0}^{t} \widetilde{g}_{q}^{\lambda}(\tau) d \tau\right)^{r}\right)^{\frac{1}{r}}+ \\
& \frac{2}{\lambda}\left(\left(2 N_{0}+1\right) 2^{\kappa}+K_{\lambda}^{1}(t)+K_{\lambda}^{2}(t)\right)\left(\sum_{q}\left(\widetilde{F}_{q}^{\lambda}(t)\right)^{r}\right)^{\frac{1}{r}},
\end{aligned}
$$

which entails the desired inequality.

Proposition 3.1 may be applied to (2.11). We end up with the following general losing estimates:

Theorem 3.2 Let $1 \leq p, p_{2}, r \leq+\infty$ and

$$
\sigma \in\left([-1]-N \min \left(\frac{1}{p_{2}}, \frac{1}{p^{\prime}}\right), 1+\frac{N}{p_{2}}\right) .
$$

There exists a $\lambda_{0}>0$ and $K=K(N)$ such that if $v_{q}, w_{q}$ and $z_{q}$ are defined as in (2.9) and satisfy

$$
v_{q} \leq v_{q}^{1}+v_{q}^{2}, \quad w_{q} \leq w_{q}^{1}+w_{q}^{2}, \quad z_{q} \leq z_{q}^{1}+z_{q}^{2},
$$


for some sequences of functions

$$
\left(v_{q}^{i}\right)_{q \in \mathbb{Z}}, \quad\left(w_{q}^{i}\right)_{q \in \mathbb{Z}} \quad \text { and } \quad\left(z_{q}^{i}\right)_{q \in \mathbb{Z}} \quad(i \in\{1,2\})
$$

verifying conditions $1,2,3$ and 4 of proposition 3.1 with $M=N_{0}=P=4$, $\sigma_{1}, \sigma_{2}$ defined in (2.10), some $\kappa \geq 0$ and some $\lambda>\lambda_{0}$, then we have

$$
\begin{aligned}
& {\left[\sum_{q}\left(\sup _{\tau \in[0, t]}\left(e^{-\lambda\left(A_{q}(\tau)+B(\tau)\right)} 2^{q \sigma}\left\|\Delta_{q} f(\tau)\right\|_{L^{p}}\right)\right)^{r}\right]^{\frac{1}{r}}} \\
& \quad \leq \frac{\lambda}{\lambda-\lambda_{0}}\left(\left\|f_{0}\right\|_{B_{p, r}^{\sigma}}+\left[\sum_{q}\left(\int_{0}^{t} e^{-\lambda\left(A_{q}(\tau)+B(\tau)\right)} 2^{q \sigma}\left\|\Delta_{q} g(\tau)\right\|_{L^{p}} d \tau\right)^{r}\right]^{\frac{1}{r}}\right) .
\end{aligned}
$$

Remark 3.3 Let us mention in passing that in the case where

$$
\nabla v \in L^{1}\left(0, T ; B_{p_{2}, \infty}^{\frac{N}{p_{2}}} \cap L^{\infty}\right) \quad \text { and } \quad \sigma<1+N / p_{2}
$$

then theorem 3.2 leads back to the inequalities of proposition 2.1 (up to a multiplicative constant in the right-hand side).

Indeed, it is only a matter of taking $v_{q}^{1}=w_{q}^{1}=z_{q}^{1}=0, \quad v_{q}^{2}=K\|\nabla v\|_{B_{p_{2}, \infty}^{\frac{N}{p_{2}} \cap L^{\infty}}}, \quad$ and $\quad w_{q}^{2}=z_{q}^{2}=K\|\nabla v\|_{B_{p_{2}, \infty}^{\frac{N}{p_{2}}}}$.

Assumption 1 is fulfilled with $R_{1}=R_{2}=R_{3}=+\infty$, one can take $\kappa=0$ in 4 and we clearly have $K_{\lambda}^{1}(t)=K_{\lambda}^{2}(t)=1$.

\subsection{Linear loss of regularity}

In this part, we aim at extending the results [1] and [6] to general Besov spaces. In the former paper, the vector field $v$ is only log-lipschitz in the space variable (which amounts to assuming that $\left\|S_{q} \nabla v(t)\right\|_{L^{\infty}} \leq(q+2) u(t)$ for some $\left.u \in L^{1}(0, T)\right)$ and estimates in Hölder spaces $C^{\sigma}$ with $\sigma \in(0,1)$ are investigated. The authors point out a loss of derivatives of order $\int_{0}^{t} u(\tau) d \tau$ at time $t$.

In the latter paper, the functional framework is more general: Besov spaces $B_{p, \infty}^{\sigma}$ with $\sigma \in(0,1)$, and the assumption on $v$ is somewhat weaker:

$$
\exists C \geq 0, \forall q \in \mathbb{N}, \frac{1}{q+2} \int_{0}^{T}\left\|S_{q} \nabla v(\tau)\right\|_{L^{\infty}} d \tau<C .
$$

We here aim at getting estimates in the same spirit in a more general framework. Our most general result reads: 
Theorem 3.4 Let $\alpha, \beta>0$ and $\sigma \in\left([-1]-\min \left(N / p_{2}, N / p^{\prime}\right)+\alpha, 1+\right.$ $\left.N / p_{2}-\beta\right)$. Let $\sigma_{1}, \sigma_{2}$ be defined as in (2.10). Assume that the following two conditions are satisfied on $[0, T]$ for some nondecreasing bounded functions $V$ and $W$ and all $q, q^{\prime} \geq-1$ :

$$
\begin{aligned}
& \text { 1. }\left|\sum_{i=0}^{+\infty} 2^{-i} \int_{0}^{t}\left(\left\|S_{q+i} \nabla v(\tau)\right\|_{L^{\infty}}-\left\|S_{q^{\prime}+i} \nabla v(\tau)\right\|_{L^{\infty}}\right) d \tau\right| \leq\left|q-q^{\prime}\right| V(t), \\
& \text { 2. }\left|\int_{0}^{t}\left(2^{\frac{q N}{p_{2}}}\left\|\Delta_{q} \nabla v(\tau)\right\|_{L^{p_{2}}}-2^{\frac{q^{\prime} N}{p_{2}}}\left\|\Delta_{q^{\prime}} \nabla v(\tau)\right\|_{L^{p_{2}}}\right) d \tau\right| \leq\left|q-q^{\prime}\right| W(t) .
\end{aligned}
$$

There exists $K=K(N)$ and $\lambda_{0}=\lambda_{0}\left(\alpha, \beta, N, p, p_{2}\right)$ such that the following inequality holds true for $\left(\mathcal{T}_{\nu}\right)$ uniformly in $\nu$ with $\sigma_{t} \stackrel{\text { def }}{=} \sigma-K \lambda(V+W)(t)$ :

$$
\begin{aligned}
& \left(\sum_{q}\left(\sup _{\tau \in[0, t]} 2^{q \sigma_{\tau}}\left\|\Delta_{q} f(\tau)\right\|_{L^{p}}\right)^{r}\right)^{\frac{1}{r}} \leq \\
& \quad \leq \frac{\lambda}{\lambda-\lambda_{0}}\left[\left\|f_{0}\right\|_{B_{p, r}^{\sigma}}+\left(\sum_{q}\left(\int_{0}^{t} 2^{q \sigma_{\tau}}\left\|\Delta_{q} g(\tau)\right\|_{L^{p}} d \tau\right)^{r}\right)^{\frac{1}{r}}\right]
\end{aligned}
$$

whenever

$$
\sigma_{1}-\lambda K(V+W)(t) \geq \alpha \quad \text { and } \quad \sigma_{2}-\lambda K(V+W)(t) \geq \beta .
$$

Proof: It stems from theorem 3.2 with an appropriate choice of $v_{q}^{i}, w_{q}^{i}$ and $z_{q}^{i}$ : let

$$
\begin{gathered}
v_{q}^{1} \stackrel{\text { def }}{=} K\left(\sum_{i=0}^{+\infty} 2^{-i}\left\|S_{q+i} \nabla v\right\|_{L^{\infty}}+\sum_{\left|q^{\prime}-q\right| \leq 4} 2^{q^{\prime} \frac{N}{p_{2}}}\left\|\Delta_{q^{\prime}} \nabla v\right\|_{L^{p_{2}}}\right), \\
w_{q}^{1} \stackrel{\text { def }}{=} z_{q}^{1} \stackrel{\text { def }}{=} K \sum_{\left|q^{\prime}-q\right| \leq 4} 2^{q^{\prime} \frac{N}{p_{2}}}\left\|\Delta_{q^{\prime}} \nabla v\right\|_{L^{p_{2}}} \quad \text { and } \quad v_{q}^{2}=w_{q}^{2}=z_{q}^{2}=0 .
\end{gathered}
$$

Choosing large enough $K=K(N)$ yields $v_{q} \leq v_{q}^{1}+v_{q}^{2}, w_{q} \leq w_{q}^{1}+w_{q}^{2}$ and $z_{q} \leq z_{q}^{1}+z_{q}^{2}$

Now, condition 1 of proposition 3.1 is trivially satisfied. So does 2 with $Q_{1}=Q_{2}=Q_{3}=-1$. Next, defining $A_{q}$ as in proposition 3.1 and taking advantage of hypotheses 1 and 2, we have (up to a change of $K$ ),

$$
\left|A_{q}(t)-A_{q^{\prime}}(t)\right| \leq K \log 2\left|q-q^{\prime}\right|(V(t)+W(t)) .
$$

Hence,

$$
K_{\lambda}^{1}(t) \leq \sum_{m>4} 2^{-m\left(\sigma_{1}-\lambda K(V+W)(t)\right)} \quad \text { and } \quad K_{\lambda}^{2}(t) \leq \sum_{m>4} 2^{-m\left(\sigma_{2}-\lambda K(V+W)(t)\right)} .
$$

Choosing $\kappa=4 \min \left(\sigma_{1}-\alpha, \sigma_{2}-\alpha\right) / \log 2$, hypothesis (3.9) ensures that conditions 3 and 4 of proposition 3.1 are satisfied for some $\lambda_{0}=\lambda_{0}\left(\alpha, \beta, N, p, p_{2}\right)$.

Then theorem 3.2 yields the desired inequality. 
Remark 3.5 Hypothesis 1 above may be replaced by the stronger following one:

$$
\left|\int_{0}^{t}\left(\left\|S_{q} \nabla v(\tau)\right\|_{L^{\infty}}-\left\|S_{q^{\prime}} \nabla v(\tau)\right\|_{L^{\infty}}\right) d \tau\right| \leq\left|q^{\prime}-q\right| V(t) .
$$

Corollary 3.6 Assume that $\nabla v \in \widetilde{L}_{T}^{1}\left(B_{p_{2}, \infty}^{\frac{N}{p_{2}}}\right)$. Then inequality (3.8) holds true with

$$
\sigma_{t}=\sigma-K \lambda\|\nabla v\|_{\widetilde{L}_{t}^{1}\left(B_{p_{2}, \infty}^{\frac{N}{p_{2}}}\right)}
$$

whenever

$$
\sigma_{1}-\lambda K\|\nabla v\|_{\widetilde{L}_{T}^{1}\left(B_{p_{2}}^{\frac{N}{p_{2}}}, \infty\right.} \geq \alpha \quad \text { and } \quad \sigma_{2}-\lambda K\|\nabla v\|_{\widetilde{L}_{T}^{1}\left(B_{p_{2}, \infty}^{\frac{N}{p_{2}}}\right)} \geq \beta .
$$

Proof: We just have to use the following inequalities (if $q^{\prime}>q$ ):

$$
\begin{aligned}
& \left|\sum_{i=0}^{+\infty} 2^{-i} \int_{0}^{t}\left(\left\|S_{q+i} \nabla v(\tau)\right\|_{L^{\infty}}-\left\|S_{q^{\prime}+i} \nabla v(\tau)\right\|_{L^{\infty}}\right) d \tau\right| \\
& \left.\quad \leq \sum_{i=0}^{+\infty} 2^{-i} \int_{0}^{t} \sum_{p=q+i}^{q^{\prime}+i-1}\left\|\Delta_{p} \nabla v(\tau)\right\|_{L^{\infty}} d \tau \leq \sum_{i=0}^{+\infty} 2^{-i}\left(q^{\prime}-q\right)\|\nabla v\|_{\widetilde{L}_{t}^{1}\left(B_{\infty, \infty}^{0}\right)}\right) \\
& \quad \leq 2\left(q^{\prime}-q\right)\|\nabla v\|_{\widetilde{L}_{t}^{1}\left(B_{\infty, \infty}^{0}\right)},
\end{aligned}
$$

and, of course, for all $q, q^{\prime} \geq-1$,

$$
\left|\int_{0}^{t}\left(2^{\frac{q N}{p_{2}}}\left\|\Delta_{q} \nabla v(\tau)\right\|_{L^{p_{2}}}-2^{\frac{q^{\prime} N}{p_{2}}}\left\|\Delta_{q^{\prime}} \nabla v(\tau)\right\|_{L^{p_{2}}}\right) d \tau\right| \leq\|\nabla v\|_{\widetilde{L}_{t}^{1}\left(B_{p_{2}, \infty}{ }^{\frac{N}{2}}\right)} .
$$

Remark 3.7 In the case $p_{2}=+\infty$, theorem 3.4 entails the result stated in $[6]$.

Remark 3.8 One can also allow for a linear growth of the dyadic blocks of the source term $g$ (like in [9], lemma 2.5). This growth will entail an additional (linear) loss of regularity. The details are left to the reader.

Actually, in the case where $\nabla v$ belongs to $\widetilde{L}_{T}^{1}\left(B_{p_{2}, \infty}^{\frac{N}{p_{2}}}\right)$ and

$$
2^{q \frac{N}{p_{2}}}\left\|\Delta_{q} \nabla v\right\|_{L_{T}^{1}\left(L^{p_{2}}\right)}
$$

is suitably small for large $q$, only the growth of $\left\|S_{q} \nabla v\right\|_{L^{\infty}}$ is responsible for the loss of regularity: 
Theorem 3.9 Let $\alpha, \beta$ and $\sigma$ satisfy the assumptions of theorem 3.4. Assume that $\nabla v$ belongs to $\widetilde{L}_{T}^{1}\left(B_{p_{2}, \infty}^{\frac{N}{p_{2}}}\right)$. There exists $\lambda_{0}=\lambda_{0}\left(\alpha, \beta, N, p, p_{2}\right)$ and $K=K(N)$ such that if $\lambda>\lambda_{0}$ and if there exists some $R \in \mathbb{N}$ such that

$$
K 2^{q \frac{N}{p_{2}}}\left\|\Delta_{q} \nabla v\right\|_{L_{T}^{1}\left(L^{p_{2}}\right)} \leq \lambda^{-1} \quad \text { for } \quad q>R
$$

then the following inequality holds true for $\left(\mathcal{T}_{\nu}\right)$ uniformly in $\nu$ :

$$
\begin{aligned}
& \left(\sum_{q}\left(\sup _{\tau \in[0, t]} e^{-\lambda W^{R}(\tau)} 2^{q \sigma_{\tau}}\left\|\Delta_{q} f(\tau)\right\|_{L^{p}}\right)^{r}\right)^{\frac{1}{r}} \\
& \quad \leq \frac{\lambda}{\lambda-\lambda_{0}}\left(\left\|f_{0}\right\|_{B_{p, r}^{\sigma}}+\left(\sum_{q}\left(\int_{0}^{t} e^{-\lambda W^{R}(\tau)} 2^{q \sigma_{\tau}}\left\|\Delta_{q} g(\tau)\right\|_{L^{p}} d \tau\right)^{r}\right)^{\frac{1}{r}}\right)
\end{aligned}
$$

with

$$
W^{R}(t) \stackrel{\text { def }}{=} \int_{0}^{t} \sup _{q \leq R+3} 2^{q \frac{N}{p_{2}}}\left\|\Delta_{q} \nabla v(\tau)\right\|_{L_{T}^{1}\left(L^{p_{2}}\right)} d \tau
$$

and

$$
\sigma_{t} \stackrel{\text { def }}{=} \sigma-K \lambda\|\nabla v\|_{\widetilde{L}_{t}^{1}\left(B_{\infty, \infty}^{0}\right)} \geq \alpha
$$

whenever

$$
\sigma_{1}-\lambda K\|\nabla v\|_{\widetilde{L}_{T}^{1}\left(B_{\infty, \infty}^{0}\right)} \geq \alpha \quad \text { and } \quad \sigma_{2}-\lambda K\|\nabla v\|_{\widetilde{L}_{T}^{1}\left(B_{\infty, \infty}^{0}\right)} \geq \beta
$$

If $\nabla v \in L^{1}\left(0, T ; B_{p_{2}, \infty}^{\frac{N}{p_{2}}}\right)$ then condition (3.10) is useless provided that $W^{R}$ has been replaced by $\|\nabla v\|_{L_{t}^{1}\left(B_{p_{2}}^{\frac{N}{p_{2}}}\right)}$.

Proof: Choose $w_{q}^{1}=z_{q}^{1}=0$,

$$
v_{q}^{1}=K\left(\sum_{q^{\prime} \leq q}\left\|\Delta_{q^{\prime}} \nabla v\right\|_{L^{\infty}}+\sum_{i \in \mathbb{N}} 2^{-i}\left\|\Delta_{q+i} \nabla v\right\|_{L^{\infty}}\right)
$$

and

$$
v_{q}^{2}=w_{q}^{2}=z_{q}^{2}=K \sum_{\left|q^{\prime}-q\right| \leq 4} 2^{q^{\prime} \frac{N}{p_{2}}}\left\|\Delta_{q^{\prime}} \nabla v\right\|_{L^{p_{2}}} .
$$

Taking advantage of the above assumptions and choosing $K=K(N)$ large enough, one can easily check that

$$
v_{q} \leq v_{q}^{1}+v_{q}^{2}, \quad w_{q} \leq w_{q}^{1}+w_{q}^{2} \quad \text { and } \quad z_{q} \leq z_{q}^{1}+z_{q}^{2} .
$$


Besides, condition 1 of proposition 3.1 is satisfied with $R_{1}=R_{2}=R_{3}=R+3$ (with the convention that $R=+\infty$ if $\nabla v \in L^{1}\left(0, T ; B_{p_{2}, \infty}^{\frac{N}{p_{2}}}\right)$ ). So does condition 2 with $Q_{1}=Q_{2}=Q_{3}=-1$. Moreover,

$$
\forall q \geq q^{\prime}, \int_{0}^{t}\left(v_{q}^{1}-v_{q^{\prime}}^{1}\right)(\tau) d \tau \leq K\left(q-q^{\prime}\right)\|\nabla v\|_{\widetilde{L}_{t}^{1}\left(B_{\infty, \infty}^{0}\right)} .
$$

so that choosing $\kappa=4 \min \left(\sigma_{1}-\alpha, \sigma_{2}-\beta\right) / \log 2$ and using (3.11) ensures conditions 3 and 4. Applying theorem 3.2 completes the proof.

In the case where the growth assumptions on the blocks are made before time integration, one can exhibit more explicit sufficient conditions for having losing estimates:

Theorem 3.10 Let $\alpha>0$ and $\sigma \in\left([-1]-\min \left(N / p_{2}, N / p^{\prime}\right)+\alpha, 1+N / p_{2}\right)$. Assume that there exist two integrable functions $u$ and $w$ such that the following conditions are satisfied on $[0, T]$ :

1. $\forall q \geq-1,\left\|S_{q} \nabla v(t)\right\|_{L^{\infty}} \leq(q+1) u(t)$

2. $\forall q \geq-1,2^{q \frac{N}{p_{2}}}\left\|\Delta_{q} \nabla v(t)\right\|_{L^{p_{2}}} \leq(q+2) w(t)$,

Let $V=\int_{0}^{t} u(\tau) d \tau$ and $W=\int_{0}^{t} w(\tau) d \tau$. There exist $\lambda_{0}=\lambda_{0}\left(\alpha, \sigma, N, p, p_{2}\right)$ and $K=K(N)$ such that whenever $\lambda>\lambda_{0}$ and $\sigma_{1}-\lambda K(V+W)(t) \geq \alpha$, we have:

$$
\begin{aligned}
& \left(\sum_{q}\left(\sup _{\tau \in[0, t]} 2^{q \sigma_{\tau}}\left\|\Delta_{q} f(\tau)\right\|_{L^{p}}\right)^{r}\right)^{\frac{1}{r}} \\
& \quad \leq \frac{\lambda}{\lambda-\lambda_{0}}\left[\left\|f_{0}\right\|_{B_{p, r}^{\sigma}}+\left(\sum_{q}\left(\int_{0}^{t} 2^{q \sigma_{\tau}}\left\|\Delta_{q} g(\tau)\right\|_{L^{p}} d \tau\right)^{r}\right)^{\frac{1}{r}}\right],
\end{aligned}
$$

with $\sigma_{t}=\sigma-K \lambda(V+W)(t)$.

Proof: Take $v_{q}^{2}=w_{q}^{2}=z_{q}^{2}=0$ and, for conveniently large $K=K(N)$,

$$
v_{q}^{1}=K(q+2)(u+w) \text { and } w_{q}^{1}=z_{q}^{1}=K(q+2) w .
$$

Then one can easily check that with the hypotheses above, we have $v_{q} \leq$ $v_{q}^{1}+v_{q}^{2}, w_{q} \leq w_{q}^{1}+w_{q}^{2}$ and $z_{q} \leq z_{q}^{1}+z_{q}^{2}$. Now, conditions of proposition 3.1 are satisfied with $Q_{i}=-1$ and $\kappa=4\left(\sigma_{1}-\alpha\right) / \log 2$ and theorem 3.2 may be applied once again while $\sigma_{1}-\lambda K(V+W)(t) \geq \alpha$ (note that $A_{q}$ is a nondecreasing sequence so that $A_{q, q^{\prime}}^{\lambda}(t) \equiv 1$ for $\left.q \geq q^{\prime}\right)$.

Remark 3.11 Note that the case $p=p_{2}=r=+\infty$ leads back to result treated in [1]. 


\subsection{Limited loss of regularity}

We now make the additional assumption that $\nabla v \in L^{1}\left(0, T ; B_{\infty, r_{2}}^{0}\right)$ for some $r_{2} \in(1,+\infty)$. The new result we get in this case is the following one:

Theorem 3.12 Assume that $\nabla v$ belongs to $\widetilde{L}_{T}^{1}\left(B_{p_{2}, \infty}^{\frac{N}{p_{2}}}\right) \cap L^{1}\left(0, T ; B_{\infty, r_{2}}^{0}\right)$ for some $r_{2} \in(1,+\infty)$. Let $\sigma \in\left([-1]-\min \left(N / p_{2}, N / p^{\prime}\right), 1+N / p_{2}\right)$ and $\epsilon \epsilon$ $\left(0, \sigma_{1} / 2\right)$ where $\sigma_{1}$ has been defined in (2.10). There exist $\lambda_{0}=\lambda_{0}\left(\sigma_{1}, \sigma_{2}\right)$ and $K=K(N)$ such that if $\lambda>\lambda_{0}$ and if there exists some $R \in \mathbb{N}$ such that

$$
K 2^{q \frac{N}{p_{2}}}\left\|\Delta_{q} \nabla v\right\|_{L_{T}^{1}\left(L^{p_{2}}\right)} \leq \lambda^{-1} \quad \text { for } \quad q>R
$$

then the following inequality holds true:

$$
\begin{aligned}
& \|f\|_{\widetilde{L}_{T}^{\infty}\left(B_{p, r}^{\sigma-\epsilon}\right)} \leq \\
& \quad \leq \frac{4^{\epsilon} \lambda e^{\lambda K W^{R}(T)}}{\lambda-\lambda_{0}} e^{\frac{(K \lambda)^{r_{2}}}{\epsilon^{r^{-1}}}\left(\int_{0}^{T}\|\nabla v(\tau)\|_{B_{\infty, r_{2}}^{0}} d \tau\right)^{r_{2}}}\left(\left\|f_{0}\right\|_{B_{p, r}^{\sigma}}+\|g\|_{\widetilde{L}_{T}^{1}\left(B_{, r}^{\sigma}\right)}\right)
\end{aligned}
$$

with $W^{R}(t) \stackrel{\text { def }}{=} \int_{0}^{t} \sup _{q \leq R+3} 2^{q \frac{N}{p_{2}}}\left\|\Delta_{q} \nabla v(\tau)\right\|_{L_{T}^{1}\left(L^{\left.p_{2}\right)}\right.} d \tau$.

If $\nabla v \in L^{1}\left(0, T ; B_{p_{2}, \infty}^{\frac{N}{p_{2}}}\right)$ then condition (3.12) may be removed and $W^{R}(T)$ has to be replaced by $\|\nabla v\|_{L_{T}^{1}\left(B_{p_{2}, \infty}^{p_{2}}\right)}$.

Proof: Once again, this is a corollary of theorem 3.2. Indeed, according to Hölder inequality,

$$
\left\|S_{q} \nabla v\right\|_{L^{\infty}} \leq(q+1)^{\eta}\|\nabla v\|_{B_{\infty, r_{2}}^{0}} \quad \text { with } \quad \eta=1-1 / r_{2}
$$

so that one can choose

$$
\begin{aligned}
& v_{q}^{1}=K(q+1)^{\eta}\|\nabla v\|_{B_{\infty, r_{2}}^{0}}, v_{q}^{2}=K\left(\sum_{q^{\prime} \geq q} 2^{q-q^{\prime}}\left\|\Delta_{q^{\prime}} \nabla v\right\|_{L^{\infty}}+\sum_{\left|q^{\prime}-q\right| \leq 4} 2^{q^{\prime} \frac{N}{p_{2}}}\left\|\Delta_{q^{\prime}} \nabla v\right\|_{L^{p_{2}}}\right) \text {, } \\
& w_{q}^{1}=z_{q}^{1}=0, \quad w_{q}^{2}=z_{q}^{2}=K \sum_{\left|q^{\prime}-q\right| \leq 4} 2^{q^{\prime} \frac{N}{p_{2}}}\left\|\Delta_{q^{\prime}} \nabla v\right\|_{L^{p_{2}}} .
\end{aligned}
$$

Condition 1 is satisfied with $R_{1}=R_{2}=R_{3}=R+3$. As $\left(v_{q}^{1}\right)_{q \geq-1}$ is a nondecreasing sequence of functions, condition 2 is fulfilled for any $Q_{1}$ (that we shall merely denote by $Q)$. Next, as $\eta \in(0,1)$, for all $Q \in \mathbb{N}$ and $q^{\prime} \geq q$, the following inequality holds:

$$
\left(q^{\prime} \wedge Q+1\right)^{\eta}-(q \wedge Q+1)^{\eta} \leq \eta\left(q^{\prime}-q\right)(Q+1)^{\eta-1} .
$$


Let $Y(t) \stackrel{\text { def }}{=} \int_{0}^{t}\|\nabla v(\tau)\|_{B_{\infty, r_{2}}^{0}} d \tau$. Up to a change of $K, A_{q}(t)=K \log 2 Y(t)$ $(q \wedge Q+1)^{\eta}$. Hence, according to inequality (3.14), we have for $q^{\prime} \geq q$,

$$
A_{q, q^{\prime}}^{\lambda}(t) \leq 2^{\lambda \eta K Y(t)\left(q^{\prime}-q\right)(Q+1)^{\eta-1}},
$$

hence

$$
K_{\lambda}^{1}(t) \leq \sum_{q^{\prime}>q+4} 2^{\left(q-q^{\prime}\right)\left(\sigma_{1}-\eta \lambda K Y(t)(Q+1)^{\eta-1}\right)}
$$

From now on, assume that

$$
Q+1 \geq\left(\frac{2 \eta \lambda K Y(t)}{\sigma_{1}}\right)^{\frac{1}{1-\eta}}
$$

Then easy computations show that $K_{\lambda}^{1}(t) \leq \sum_{m>4} 2^{-p \frac{\sigma_{1}}{2}}=2^{-5 \frac{\sigma_{1}}{2}} /\left(1-2^{-\frac{\sigma_{1}}{2}}\right)$. As $A_{q}$ is nondecreasing, we readily get $K_{\lambda}^{2}(t) \leq 2^{-5 \sigma_{2}} /\left(1-2^{-\sigma_{2}}\right)$.

Now, if we take $\kappa=2 \sigma_{1} / \log 2$, inequality (3.14) and the above computations show that conditions 3 and 4 are fulfilled for some $\lambda_{0}=\lambda_{0}\left(\sigma_{1}, \sigma_{2}\right)$. Hence, theorem 3.2 yields

$$
\begin{aligned}
& {\left[\sum_{q}\left(\sup _{[0, t]}\left(e^{-K \lambda W^{R}(\tau)} 2^{q \epsilon-[(q \wedge Q)+1]^{\eta} K \lambda Y(\tau)} 2^{q(\sigma-\epsilon)}\left\|\Delta_{q} f(\tau)\right\|_{L^{p}}\right)\right)^{r}\right]^{\frac{1}{r}}} \\
& \leq \frac{\lambda}{\lambda-\lambda_{0}}\left(\left\|f_{0}\right\|_{B_{p, r}^{\sigma}+}\left[\sum_{q}\left(\int_{0}^{t} e^{-K \lambda W^{R}(\tau)} 2^{-[(q \wedge Q)+1]^{\eta} K \lambda Y(\tau)} 2^{q \sigma}\left\|\Delta_{q} g(\tau)\right\|_{L^{p}} d \tau\right)^{r}\right]^{\frac{1}{r}}\right) .
\end{aligned}
$$

Now, getting the desired inequality in theorem 3.12 amounts to making a convenient choice of $Q$. Indeed, denoting $C=\eta \lambda K \epsilon^{-1} Y(T)$, we have

$$
\forall x>0, \epsilon(x+1)-C x^{\eta} \geq \epsilon+\left(\frac{C}{\epsilon^{\eta}}\right)^{\frac{1}{1-\eta}}\left(\eta^{\frac{1}{1-\eta}}-\eta^{\frac{\eta}{1-\eta}}\right) \geq \epsilon-\left(\frac{C \eta^{\eta}}{\epsilon^{\eta}}\right)^{\frac{1}{1-\eta}} .
$$

Therefore

$$
\begin{aligned}
q \epsilon-K \lambda Y(t) & (1+q \wedge Q)^{\eta} \geq \\
& \geq \begin{cases}\epsilon-\left(\frac{K \lambda Y(t) \eta^{\eta}}{\epsilon^{\eta}}\right)^{\frac{1}{1-\eta}} & \text { if } \quad q \geq Q, \\
-\epsilon-K \lambda Y(t)(1+Q)^{\eta} & \text { if } \quad-1 \leq q<Q .\end{cases}
\end{aligned}
$$

Assuming that $0<\epsilon \leq \sigma_{1} / 2$ and choosing

$$
Q \stackrel{\text { def }}{=}\left[\left(\frac{\eta K \lambda Y(T)}{\epsilon}\right)^{\frac{1}{1-\eta}}\right]
$$


condition (3.16) is fulfilled on $[0, T]$, and we have, according to (3.17)

$$
\left.2^{q \epsilon-[(q \wedge Q)+1]^{\eta} K \lambda Y(T)} \leq 2^{2 \epsilon\left(1+\left(\lambda \epsilon^{-1} Y(T)\right)^{\frac{1}{1-\eta}}\right.}\right) .
$$

This implies inequality (3.13).

Actually, estimates with limited loss of regularity may be proved under the weaker additional assumption that $\nabla v \in \widetilde{L}_{T}^{1}\left(B_{\infty, r_{2}}^{0}\right)$ for some $r_{2} \in$ $(1,+\infty)$ :

Theorem 3.13 Assume that $\nabla v$ belongs to $\widetilde{L}_{T}^{1}\left(B_{p_{2}, \infty}^{\frac{N}{p_{2}}}\right) \cap \widetilde{L}_{T}^{1}\left(B_{\infty, r_{2}}^{0}\right)$ for some $r_{2} \in(1,+\infty)$. Let $\sigma \in\left([-1]-\min \left(N / p_{2}, N / p^{\prime}\right), 1+N / p_{2}\right)$ and $\epsilon \in\left(0, \sigma_{1}\right)$. There exist $\lambda_{0}=\lambda_{0}\left(\sigma_{1}, \sigma_{2}\right)$ and $K=K(N)$ such that if $\lambda>\lambda_{0}$ and if there exists some $R \in \mathbb{N}$ such that (3.12) is fulfilled, then the following inequality holds true:

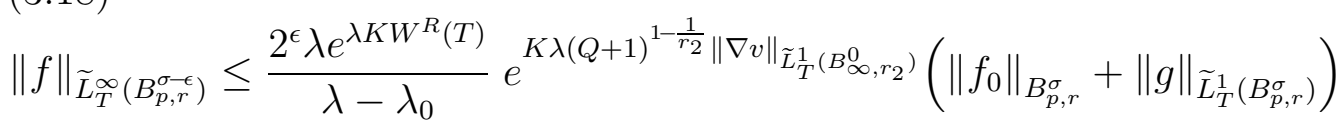

with $Q$ such that $\sup _{q \geq Q}\left\|\nabla \Delta_{q} v\right\|_{L_{T}^{1}\left(L^{\infty}\right)} \leq \epsilon / K \lambda$ and $W^{R}(t)$ defined as in theorem 3.12 (with the usual change if $\nabla v \in L^{1}\left(0, T ; B_{p_{2}, \infty}^{\frac{N}{p_{2}}}\right)$ ).

Proof: Define $v_{q}^{2}, w_{q}^{2}, z_{q}^{2}, w_{q}^{1}, z_{q}^{1}$ as previously, and $v_{q}^{1} \stackrel{\text { def }}{=} K \sum_{q^{\prime} \leq q-1}\left\|\Delta_{q} \nabla v\right\|_{L^{\infty}}$. As for $q^{\prime} \geq q$, we have (up to a change of $K$ )

$$
\int_{0}^{T}\left(v_{q^{\prime}}^{1}(t)-v_{q}^{1}(t)\right) d t \leq K \log 2\left(q^{\prime}-q\right) \sup _{q^{\prime \prime} \geq q}\left\|\Delta_{q} \nabla v\right\|_{L_{T}^{1}\left(L^{\infty}\right)},
$$

we easily get by choosing $Q_{1}=Q$,

$$
\forall q^{\prime} \geq q, A_{q, q^{\prime}}^{\lambda} \leq 2^{\epsilon\left(q^{\prime}-q\right)} .
$$

Hence condition 3 is satisfied. So does 4 with $\kappa=2 \sigma_{1} / \log 2$. Therefore the general inequality stated in theorem 3.2 holds true with $B(t)=W^{R}(t)$ and $A_{q}(t)=K \int_{0}^{t} \sum_{p \leq q \wedge Q-1}\left\|\Delta_{q} \nabla v(\tau)\right\|_{L^{\infty}} d \tau$. Now, according to Hölder inequality and the definition of $Q$, we have

$$
\begin{aligned}
K \log 2 \lambda \int_{0}^{t} \sum_{p \leq q \wedge Q-1} & \left\|\Delta_{q} \nabla v(\tau)\right\|_{L^{\infty}} d \tau \\
& \leq K \log 2 \lambda(Q+1)^{1-\frac{1}{r_{2}}}\|\nabla v\|_{\widetilde{L}_{T}^{1}\left(B_{\infty, r_{2}}^{0}\right)}+\epsilon \max (0, q-Q),
\end{aligned}
$$

whence for all $t \in[0, T]$ and $q \geq-1$,

$$
e^{\lambda A_{q}(t)} 2^{-q \epsilon} \leq e^{K \lambda(Q+1)^{1-\frac{1}{r_{2}}}\|\nabla v\|_{\widetilde{L}_{T}^{1}\left(B_{\infty, r_{2}}^{0}\right)}}
$$

This yields inequality (3.18). 


\section{A. Appendix}

We here give the proof of lemma 2.2. In order to show that only the gradient part of $v$ is involved in the estimates, it is convenient to split $v$ into low and high frequencies: $v=\Delta_{-1} v+\widetilde{v}$. Obviously, there exists a constant $C$ such that

$$
\forall r \in[1,+\infty],\left\|\Delta_{-1} \nabla v\right\|_{L^{r}} \leq C\|\nabla v\|_{L^{r}} \text { and }\|\nabla \widetilde{v}\|_{L^{r}} \leq C\|\nabla v\|_{L^{r}} .
$$

Since there exists a $R>0$ so that for all $t \in[0, T]$, Supp $\mathcal{F} \widetilde{v}(t) \cap B(0, R)=\emptyset$, Bernstein inequality holds true for $\Delta_{q} \widetilde{v}$ even for $q=-1$, namely

$$
\forall q \geq-1,\left\|\Delta_{q} \nabla \widetilde{v}\right\|_{L^{p}} \approx 2^{q}\left\|\Delta_{q} \widetilde{v}\right\|_{L^{p}} .
$$

Let us define the paraproduct between two distributions according to J.-M. Bony in [2]:

$$
T_{f} g \stackrel{\text { def }}{=} \sum_{q \in \mathbb{N}} S_{q-1} f \Delta_{q} g
$$

Denoting

$$
R(f, g) \stackrel{\text { def }}{=} \sum_{q \geq-1} \Delta_{q} f \widetilde{\Delta}_{q} g \quad \text { with } \quad \widetilde{\Delta_{q}} g \stackrel{\text { def }}{=}\left(\Delta_{q-1}+\Delta_{q}+\Delta_{q+1}\right) g
$$

we have the following so-called Bony's decomposition:

$$
f g=T_{f} g+T_{g} f+R(f, g) .
$$

Now, we have

$$
\begin{aligned}
R_{q} & =S_{q+1} v \cdot \nabla \Delta_{q} f-\Delta_{q}(v \cdot \nabla f), \\
& =\left[\widetilde{v}^{j}, \Delta_{q}\right] \partial_{j} f+\left[\Delta_{-1} v^{j}, \Delta_{q}\right] \partial_{j} f+\left(S_{q+1} v-v\right) \cdot \nabla \Delta_{q} f,
\end{aligned}
$$

where the summation convention on repeated indices is understood.

Hence, taking advantage of Bony's decomposition, we end up with $R_{q}=$ $\sum_{i=1}^{7} R_{q}^{i}$ where

$$
\begin{aligned}
R_{q}^{1} & =\left[T_{\widetilde{v}^{j}}, \Delta_{q}\right] \partial_{j} f, \\
R_{q}^{2} & =T_{\partial_{j} \Delta_{q} f} \widetilde{v}^{j}, \\
R_{q}^{3} & =-\Delta_{q} T_{\partial_{j}} \widetilde{v}^{j}, \\
R_{q}^{4} & =\partial_{j} R\left(\widetilde{v}^{j}, \Delta_{q} f\right)-\partial_{j} \Delta_{q} R\left(\widetilde{v}^{j}, f\right), \\
R_{q}^{5} & =\Delta_{q} R(\operatorname{div} \widetilde{v}, f)-R\left(\operatorname{div} \widetilde{v}, \Delta_{q} f\right), \\
R_{q}^{6} & =\left(S_{q+1} v-v\right) \cdot \nabla \Delta_{q} f, \\
R_{q}^{7} & =\left[\Delta_{-1} v^{j}, \Delta_{q}\right] \partial_{j} f .
\end{aligned}
$$

In the following computations, the constant $K$ depends only on $N$. 
Bounds for $2^{q \sigma}\left\|R_{q}^{1}\right\|_{L^{p}}$ :

By virtue of (1.2), we have

$$
R_{q}^{1}=\sum_{\left|q-q^{\prime}\right| \leq 4}\left[S_{q^{\prime}-1} \widetilde{v}^{j}, \Delta_{q}\right] \partial_{j} \Delta_{q^{\prime}} f .
$$

On the other hand,

$\left[S_{q^{\prime}-1} \widetilde{v}^{j}, \Delta_{q}\right] \partial_{j} \Delta_{q^{\prime}} f(x)=\int h(y)\left[S_{q^{\prime}-1} \widetilde{v}^{j}(x)-S_{q^{\prime}-1} \widetilde{v}^{j}\left(x-2^{-q} y\right)\right] \partial_{j} \Delta_{q^{\prime}} f\left(x-2^{-q} y\right) d y$ so that applying first order Taylor's formula, convolution inequalities and (A.1) yields

$$
2^{q \sigma}\left\|R_{q}^{1}\right\|_{L^{p}} \leq K \sum_{\left|q^{\prime}-q\right| \leq 4}\left\|S_{q^{\prime}-1} \nabla v\right\|_{L^{\infty}} 2^{q^{\prime} \sigma}\left\|\Delta_{q^{\prime}} f\right\|_{L^{p}}
$$

Bounds for $2^{q \sigma}\left\|R_{q}^{2}\right\|_{L^{p}}$ :

By virtue of (1.2), we have

$$
R_{q}^{2}=\sum_{q^{\prime} \geq q-3} S_{q^{\prime}-1} \partial_{j} \Delta_{q} f \Delta_{q^{\prime}} \widetilde{v}^{j} .
$$

Hence, using inequalities (A.1) and (A.2) yields

$$
2^{q \sigma}\left\|R_{q}^{2}\right\|_{L^{p}} \leq K \sum_{q^{\prime} \geq q-3} 2^{q-q^{\prime}} 2^{q \sigma}\left\|\Delta_{q} f\right\|_{L^{p}}\left\|\Delta_{q^{\prime}} \nabla v\right\|_{L^{\infty}} .
$$

Bounds for $2^{q \sigma}\left\|R_{q}^{3}\right\|_{L^{p}}$ :

One proceeds as follows:

$$
\begin{aligned}
R_{q}^{3} & =-\sum_{\left|q^{\prime}-q\right| \leq 4} \Delta_{q}\left(S_{q^{\prime}-1} \partial_{j} f \Delta_{q^{\prime}} \widetilde{v}^{j}\right), \\
& =-\sum_{\substack{\left|q^{\prime}-q\right| \leq 4 \\
q^{\prime \prime} \leq q^{\prime}-2}} \Delta_{q}\left(\Delta_{q^{\prime \prime}} \partial_{j} f \Delta_{q^{\prime}} \widetilde{v}^{j}\right) .
\end{aligned}
$$

Therefore, denoting $1 / p_{1}=1 / p-1 / p_{2}$ and taking advantage of (A.1) and (A.2),

$$
\begin{aligned}
& 2^{q \sigma}\left\|R_{q}^{3}\right\|_{L^{p}} \leq K \sum_{\substack{\left|q^{\prime}-q\right| \leq 4 \\
q^{\prime \prime} \leq q^{\prime}-2}} 2^{q \sigma}\left\|\Delta_{q^{\prime \prime}} \partial_{j} f\right\|_{L^{p_{1}}}\left\|\Delta_{q^{\prime}} \widetilde{v}^{j}\right\|_{L^{p_{2}}}, \\
& \text { A.7) } \quad K \sum_{\substack{\left|q^{\prime}=q\right| \leq 4 \\
q^{\prime \prime} \leq q^{\prime}-2}} 2^{\left(q-q^{\prime \prime}\right)\left(\sigma-1-\frac{N}{p_{2}}\right)} 2^{q^{\prime \prime} \sigma}\left\|\Delta_{q^{\prime \prime}} f\right\|_{L^{p}} 2^{q^{\prime} \frac{N}{p_{2}}}\left\|\Delta_{q^{\prime}} \nabla v\right\|_{L^{p_{2}}} .
\end{aligned}
$$

Note that, starting from (A.5), one can alternately get

$$
2^{q \sigma}\left\|R_{q}^{3}\right\|_{L^{p}} \leq 16^{|\sigma|} K \sum_{\left|q^{\prime}-q\right| \leq 4}\left\|\nabla S_{q^{\prime}-1} f\right\|_{L^{p_{1}}} 2^{q^{\prime}(\sigma-1)}\left\|\Delta_{q^{\prime}} \nabla v\right\|_{L^{p_{2}}} .
$$


Bounds for $2^{q \sigma}\left\|R_{q}^{4}\right\|_{L^{p}}$ :

$$
R_{q}^{4}=\underbrace{\sum_{\left|q^{\prime}-q\right| \leq 2} \partial_{j}\left(\Delta_{q^{\prime}} \widetilde{v}^{j} \Delta_{q} \widetilde{\Delta}_{q^{\prime}} f\right)}_{R_{q}^{4,1}}-\underbrace{\sum_{q^{\prime} \geq q-3} \partial_{j} \Delta_{q}\left(\Delta_{q^{\prime}} \widetilde{v}^{j} \widetilde{\Delta}_{q^{\prime}} f\right)}_{R_{q}^{4,2}} .
$$

For the first term, we merely have (by virtue of (A.2)),

$$
2^{q \sigma}\left\|R_{q}^{4,1}\right\|_{L^{p}} \leq 4^{|\sigma|} K \sum_{\left|q^{\prime}-q\right| \leq 2}\left\|\Delta_{q^{\prime}} \nabla \widetilde{v}\right\|_{L^{\infty}} 2^{q^{\prime} \sigma}\left\|\widetilde{\Delta}_{q^{\prime}} f\right\|_{L^{p}} .
$$

For $R_{q}^{4,2}$, we proceed differently depending on the value of $1 / p+1 / p_{2}$.

First case: $1 / p_{3} \stackrel{\text { def }}{=} 1 / p+1 / p_{2} \leq 1$ :

$$
\begin{aligned}
2^{q \sigma}\left\|R_{q}^{4,2}\right\|_{L^{p}} & \leq K \sum_{q^{\prime} \geq q-3} 2^{q(1+\sigma)} 2^{q\left(\frac{N}{p_{3}}-\frac{N}{p}\right)}\left\|\Delta_{q^{\prime}} \widetilde{v} \widetilde{\Delta}_{q^{\prime}} f\right\|_{L^{p_{3}}}, \\
& \leq K \sum_{q^{\prime} \geq q-3} 2^{q(1+\sigma)} 2^{q \frac{N}{p_{2}}}\left\|\Delta_{q^{\prime}} \widetilde{v}\right\|_{L^{p_{2}}}\left\|\widetilde{\Delta}_{q^{\prime}} f\right\|_{L^{p}}, \\
& \leq K \sum_{q^{\prime} \geq q-3} 2^{\left(q-q^{\prime}\right)\left(1+\frac{N}{p_{2}}+\sigma\right)} 2^{q^{\prime} \frac{N}{p_{2}}}\left\|\nabla \Delta_{q^{\prime}} \widetilde{v}\right\|_{L^{p_{2}}} 2^{q^{\prime} \sigma}\left\|\widetilde{\Delta}_{q^{\prime}} f\right\|_{L^{p}} .
\end{aligned}
$$

Second case: $1 / p+1 / p_{2}>1$ :

Taking $p_{2}=p^{\prime}$ in the above computations yields

$$
\begin{aligned}
2^{q \sigma}\left\|R_{q}^{4,2}\right\|_{L^{p}} & \leq K \sum_{q^{\prime} \geq q-3} 2^{\left(q-q^{\prime}\right)\left(1+\frac{N}{p^{\prime}}+\sigma\right)} 2^{q^{\prime} \frac{N}{p^{\prime}}}\left\|\nabla \Delta_{q^{\prime}} \widetilde{v}\right\|_{L^{p^{\prime}}} 2^{q^{\prime} \sigma}\left\|\widetilde{\Delta}_{q^{\prime}} f\right\|_{L^{p}}, \\
& \leq K \sum_{q^{\prime} \geq q-3} 2^{\left(q-q^{\prime}\right)\left(1+\frac{N}{p^{\prime}}+\sigma\right)} 2^{q^{\prime} \frac{N}{p_{2}}}\left\|\nabla \Delta_{q^{\prime}} \widetilde{v}\right\|_{L^{p_{2}}} 2^{q^{\prime} \sigma}\left\|\widetilde{\Delta}_{q^{\prime}} f\right\|_{L^{p}} .
\end{aligned}
$$

In view of (A.1) and (A.9), we conclude that

$$
\begin{array}{ll}
\text { (A.10) } & 2^{q \sigma}\left\|R_{q}^{4}\right\|_{L^{p}} \leq \\
& \leq 4^{|\sigma|} K \sum_{q^{\prime} \geq q-3} 2^{\left(q-q^{\prime}\right)\left(1+\sigma+N \min \left(\frac{1}{p_{2}}, \frac{1}{p^{\prime}}\right)\right)} 2^{q^{\prime} \frac{N}{p_{2}}}\left\|\Delta_{q^{\prime}} \nabla v\right\|_{L^{p_{2}}} 2^{q^{\prime} \sigma}\left\|\widetilde{\Delta}_{q^{\prime}} f\right\|_{L^{p}} .
\end{array}
$$

Bounds for $2^{q \sigma}\left\|R_{q}^{5}\right\|_{L^{p}}$ :

Similar computations yield

$$
\begin{array}{ll}
\text { (A.11) } & 2^{q \sigma}\left\|R_{q}^{5}\right\|_{L^{p}} \leq \\
& \leq 4^{|\sigma|} K \sum_{q^{\prime} \geq q-3} 2^{\left(q-q^{\prime}\right)\left(\sigma+N \min \left(\frac{1}{p_{2}}, \frac{1}{p^{\prime}}\right)\right)} 2^{q^{\prime} \frac{N}{p_{2}}}\left\|\Delta_{q^{\prime}} \operatorname{div} v\right\|_{L^{p_{2}}} 2^{q^{\prime} \sigma}\left\|\widetilde{\Delta}_{q^{\prime}} f\right\|_{L^{p}} .
\end{array}
$$


Bounds for $2^{q \sigma}\left\|R_{q}^{6}\right\|_{L^{p}}$ :

Since $R_{q}^{6}=-\sum_{q^{\prime} \geq q+1} \Delta_{q^{\prime}} v \cdot \nabla \Delta_{q} f$, we have, by virtue of Bernstein inequality (note that $q^{\prime} \geq 0$ in the summation),

$$
2^{q \sigma}\left\|R_{q}^{6}\right\|_{L^{p}} \leq K \sum_{q^{\prime} \geq q} 2^{q-q^{\prime}}\left\|\Delta_{q^{\prime}} \nabla v\right\|_{L^{\infty}} 2^{q \sigma}\left\|\Delta_{q} f\right\|_{L^{p}}
$$

Bounds for $2^{q \sigma}\left\|R_{q}^{7}\right\|_{L^{p}}$ :

As $R_{q}^{7}=\sum_{\left|q^{\prime}-q\right| \leq 1}\left[\Delta_{q}, \Delta_{-1} v\right] \cdot \nabla \Delta_{q^{\prime}} f$, the first order Taylor formula yields

$$
2^{q \sigma}\left\|R_{q}^{7}\right\|_{L^{p}} \leq 2^{|\sigma|} K \sum_{\left|q^{\prime}-q\right| \leq 1}\left\|\nabla \Delta_{-1} v\right\|_{L^{\infty}} 2^{q^{\prime} \sigma}\left\|\Delta_{q^{\prime}} f\right\|_{L^{p}} .
$$

Combining inequalities (A.3), (A.4), (A.7) or (A.8), (A.10), (A.11), (A.12), and (A.13), we end up with the desired estimate for $R_{q}$.

Remark A.1 Straightforward modifications in the estimates for $R_{q}^{3}, R_{q}^{4}, R_{q}^{5}$ show that in the special case where $f=v$, the following estimate holds true:

$$
\begin{aligned}
2^{q \sigma}\left\|R_{q}\right\|_{L^{p}} \lesssim & \sum_{\left|q^{\prime}-q\right| \leq 4}\left\|S_{q^{\prime}+1} \nabla v\right\|_{L^{\infty}} 2^{q^{\prime} \sigma}\left\|\Delta_{q^{\prime}} v\right\|_{L^{p}}+\sum_{q^{\prime} \geq q-3} 2^{q-q^{\prime}} 2^{q \sigma}\left\|\Delta_{q^{\prime}} v\right\|_{L^{p}}\left\|\Delta_{q^{\prime}} \nabla v\right\|_{L^{\infty}} \\
& \quad+\sum_{\substack{q^{\prime} \geq q-3 \\
\left|q^{\prime}-q^{\prime \prime}\right| \leq 1}} 2^{\left(q-q^{\prime}\right) \sigma}\left(2^{q-q^{\prime}}\left\|\Delta_{q^{\prime}} \nabla v\right\|_{L^{\infty}}+\left\|\Delta_{q^{\prime}} \operatorname{div} v\right\|_{L^{\infty}}\right) 2^{q^{\prime \prime} \sigma}\left\|\Delta_{q^{\prime \prime}} v\right\|_{L^{p}} .
\end{aligned}
$$

\section{References}

[1] Bahouri, H. And Chemin, J.-Y.: Équations de transport relatives à des champs de vecteurs non lipschitziens et mécanique des fluides. Arch. Rational Mech. Anal. 127 (1994), 159-181.

[2] Bony, J.-M.: Calcul symbolique et propagation des singularités pour les équations aux dérivées partielles non linéaires. Ann. Sci. École Norm. Sup. (4) 14 (1981), 209-246.

[3] Chemin, J.-Y.: Perfect incompressible fluids. Oxford Lecture Series in Mathematics and its Applications, 14. The Clarendon Press, Oxford University Press, New York, 1998.

[4] Chemin, J.-Y.: Théorèmes d'unicité pour le système de Navier-Stokes tridimensionnel. J. Anal. Math. 77 (1999), 27-50.

[5] Chemin, J.-Y. And Lerner, N.: Flot de champs de vecteurs non lipschitziens et équations de Navier-Stokes. J. Differential Equations 121 (1995), 314-328.

[6] Chemin, J.-Y. And Masmoudi, N.: About lifespan of regular solutions of equations related to viscoelastic fluids. SIAM J. Math. Anal. 33 (2001), $84-112$. 
[7] Colombini, F. And Lerner, N.: Uniqueness of continuous solutions for BV vector fields. Duke Math. J. 111 (2002), 357-384.

[8] Colombini, F. and Lerner, N.: Uniqueness of $L^{\infty}$ solutions for a class of conormal BV vector fields. In Geometric analysis of PDE and several complex variables, 133-156. Contemp. Math. 368, Amer. Math. Soc., Providence, RI, 2005.

[9] Danchin, R.: Évolution d'une singularité de type cusp dans une poche de tourbillon. Rev. Mat. Iberoamericana 16 (2000), 281-329.

[10] Danchin, R.: A few remarks on the Camassa-Holm equation. Differential Integral Equations 14 (2001), 953-988.

[11] Danchin, R.: Local and global well-posedness results for flows of inhomogeneous viscous fluids. Adv. Differential Equations 9 (2004), 353-386.

[12] Danchin, R.: Local theory in critical spaces for compressible viscous and heat-conductive gases. Comm. Partial Differential Equations 26 (2001), 1183-1233, and Erratum, 27 (2002), 2531-2532.

[13] Desjardins, B.: A few remarks on ordinary differential equations. Comm. Partial Differential Equations 21 (1996), 1667-1703.

[14] Desjardins, B.: Linear transport equations with initial values in Sobolev spaces and application to the Navier-Stokes equations. Differential Integral Equations 10 (1997), 577-586.

[15] Desjardins, B.: Regularity results for two-dimensional flows of multiphase viscous fluids. Arch. Rational Mech. Anal. 137 (1997), 135-158.

[16] Dutrifoy, A.: Precise regularity results for the Euler equations. J. Math. Anal. Appl. 282 (2003), 177-200.

[17] Planchon, F.: An extension of the Beale-Kato-Majda criterion for the Euler equations. Comm. Math. Phys. 232 (2003), 319-326.

[18] Runst, T. And Sickel, W.: Sobolev spaces of fractional order, Nemytskij operators, and nonlinear partial differential equations. De Gruyter Series in Nonlinear Analysis and Applications, 3. Walter de Gruyter, Berlin, 1996.

[19] Vishik, M.: Incompressible flows of an ideal fluid with vorticity in borderline spaces of Besov type. Ann. Sci. École Norm. Sup. 32 (1999), 769-812.

Recibido: 11 de junio de 2003

Revisado: 6 de febrero de 2004

Raphaël Danchin

Centre de Mathématiques

Université Paris 12

61 avenue du Général de Gaulle 94010 Créteil Cedex, France danchin@univ-paris12.fr 